University of Windsor

Scholarship at UWindsor

Winter 2010

\title{
Social responsibility allocation in two-echelon supply chains: Insights from wholesale price contracts
}

Debing $\mathrm{Ni}$

Kevin W. Li Dr.

University of Windsor

Xiaowo Tang

Follow this and additional works at: https://scholar.uwindsor.ca/odettepub

Part of the Business Commons

\section{Recommended Citation}

Ni, Debing; Li, Kevin W. Dr.; and Tang, Xiaowo. (2010). Social responsibility allocation in two-echelon supply chains: Insights from wholesale price contracts. European Journal of Operational Research, 207 (3), 1269-1279.

https://scholar.uwindsor.ca/odettepub/61

This Article is brought to you for free and open access by the Odette School of Business at Scholarship at UWindsor. It has been accepted for inclusion in Odette School of Business Publications by an authorized administrator of Scholarship at UWindsor. For more information, please contact scholarship@uwindsor.ca. 


\section{Social Responsibility Allocation in Two-echelon Supply Chains: Insights} from Wholesale Price Contracts

\author{
Debing $\mathrm{Ni}^{1}$, Kevin W. $\mathrm{Li}^{2 *}$, Xiaowo Tang ${ }^{1}$
}

1 School of Management and Economics, University of Electronic Science and Technology of China, Chengdu, Sichuan, P. R. China, 610054

2 Odette School of Business, University of Windsor, Windsor, Ontario, Canada, N9B 3P4

Abstract: Corporate social responsibility (CSR) is defined as corporate activities and their impacts on different social groups. In this paper, CSR is considered in a two-echelon supply chain consisting of an upstream supplier and a downstream firm that are bound by a wholesale price contract. CSR performance (the outcome of CSR conduct) of the whole supply chain is gauged by a global variable and the associated cost of achieving this CSR performance is only incurred by the supplier with an expectation of being shared with the downstream firm via the wholesale price contract. As such, the key issue is to determine who should be allocated as the responsibility holder with the right of offering the contract and how this right should be appropriately restricted. Game-theoretical analyses are carried out on six games, resulting from different interaction schemes between the supplier and the firm, to derive their corresponding equilibriums. Comparative institutional analyses are then conducted to determine the optimal social responsibility allocations based on both economic and CSR performance criteria. Main results are furnished in a series of propositions and their implications to the real-world business practice are discussed. The key findings are threefold: Under the current model settings, (1) the optimal allocation scheme is to assign the supplier as the responsibility holder with appropriate restrictions on the corresponding rights to determine the wholesale price; (2) Inherent conflict exists between the economic and CSR performance criteria and, hence, the two maxima cannot be achieved simultaneously; (3) Although integrative channel profit is not attainable, the systemwide profit will be improved by implementing optimal social responsibility allocation schemes.

Keywords: Supply chain management; corporate social responsibility; wholesale price contracts; equilibrium.

\section{Introduction}

Corporate social responsibility (CSR) is defined as corporate activities and their impacts on

\footnotetext{
* Correspondence author, phone: 519-253-3000 ext 3456, fax: 519-973-7073, e-mail: kwli@uwindsor.ca
} 
different social groups, including human rights, environment protection (e.g. recycling used

31 product), pollutant emission control, philanthropy, to name a few (Cater and Jennings 2002).

32 CSR has been receiving considerable attention in the academic community, from the CSR

33 construct in the 1950s (Bowen 1953) to empirical investigations on the relationship between

34 CSR and corporate financial performance (CFP) ${ }^{1}$ and, then, to formal modeling of CSR (Baron

35 2001, 2007, Calveras et al. 2007, Giovanni and Giacinta 2007). In recent years, with the

36 continued trend of globalization, the research on supply chain management has enabled firms to

37 improve their profitability by fostering partnership with other members in their supply chain

38 systems. While firms enjoy improved efficiency, pressures are also accumulating for socially and

39 environmentally responsible supply chain practice (Linton et al. 2007). For instance, many

40 leading brands such as Nike, GAP, Adidas, and McDonalds have been urged to incorporate social

41 responsibility into their supply chains (Amaeshi et al. 2008). In response to this pressure, many

42 supply chain primary firms have introduced codes of conduct to ensure their partners' business

43 practices to be socially responsible. However, World Bank (2003) reports that implementing

44 codes of conduct is challenged by a plethora of individual CSR codes, the effectiveness of the 45 top-down CSR strategies and insufficient understanding of business benefits.

46 Note that even if a lobby group (e.g., non-governmental organizations) for social 47 responsibility may only target a particular firm in a supply chain, the pressure can be easily 48 propagated to other members in the system through their business transactions. Therefore, it is 49 necessary to extend the traditional CSR beyond a single firm's boundary and consider it within a 50 supply chain context (Davis et al. 1997; Mamic 2005). Recent research has started to model 51 social responsibility in supply chain operations. For instance, Savaskan et al. (2004) develop a 52 model for closed-loop supply chains with product remanufacturing and identify an appropriate 53 supply chain structure for original equipment manufacturers. Crutz (2008) introduces a dynamic 54 multi-criteria decision-making framework for modeling and analyzing the equilibrium of supply 55 chain network with environmental responsibility where environmental (social) responsibility is 56 assumed to have no direct impact on market demand and the allocation of environmental (social) 57 responsibility is not explicitly considered. In Hsueh and Chang's (2008) three-tier (manufacturer, 58 distributor and retailer) supply chain network model, the allocation of CSR for system-wide 59 optimization is captured by additional monetary transfers (via an enforceable agreement), and

\footnotetext{
${ }^{1}$ See Orlitzky et al. (2003) for a meta-analysis and Margolis and Walsh (2001) for a survey on empirical studies.
} 
this treatment allows for the assumption of each decentralized manufacturer's marginal

61 production cost to be the same as that in the centralized network. For empirical studies, Carter et

62 al. (2000) show that environmental purchasing has significant impacts on both income and cost.

63 Cater and Jennings (2002) find a positive relationship between CSR and supplier performance.

Although these studies attempt to incorporate social responsibility into supply chain management, the allocation of social reasonability has not emerged as a main focus, whereas it is a critical issue for supply chain members to collaboratively manage the extended CSR. As 67 OECD (2001) states, "allocating responsibility and determining who is the producer [the responsibility holder] are two of the most important [EPR, Extended Producer Responsibility] policy issues." On the one hand, the principles of corporate legal personality and separate existence of corporations naturally reject the extension of the responsibility of one member to any others. In this respect, all members in a supply chain are responsible for only their own actions. But on the other hand, the stakeholder theory (Freeman 1984) argues that each supply chain member shares the responsibility for other members' actions. Now a natural question is how to handle social responsibility in the context of a supply chain: is social responsibility independent for individual firms or shared among different entities? This article follows the second argument and treats social responsibility as shared obligations among supply chain partners. In this case, it is crucial to know how the responsibility is allocated among the firms. Otherwise, unclear allocation is likely to lead to the "tragedy of the commons" and result in lower supply chain efficiency. As an example, Amaeshi et al. (2008) suggest that the more

80 powerful member in a firm-supplier relationship should bear the responsibility to influence the 81 less powerful one(s).

This research aims to address the social responsibility allocation problem in a two-echelon 83 supply chain under wholesale price contracts. The basic settings of the model are outlined as 84 follows: a two-echelon supply chain consists of two members, an upstream supplier (S) and a 85 downstream firm (F). The investment in CSR always incurs by the supplier, which provides a 86 global measurement of the CSR performance for the supply chain and is assumed to be 87 independent of the production quantity ${ }^{2}$. This cost is then shared with the firm via a wholesale

\footnotetext{
${ }^{2}$ It is widely observed that the main target in supply chain CSR is at the supplier side. For example, Nike and its subcontractors are often accused of inhumane labor and business practices in its Asian manufacturing facilities (Amaeshi et al. 2008). As the largest specialty apparel retailer, GAP admits to the charge of its substandard working conditions in as many as 3000 of its factories (Merrick 2004). Moreover, CSR activity such as human rights and philanthropy are almost irrelevant to production quantity. Xiao and Yang (2008) and Tsay and Agrawal (2000) make this same assumption in their research as well.
} 
88 price contract $^{3}$ that is an increasing function of CSR investment by the supplier ${ }^{4}$. Three power 89 structures are entertained, $\mathrm{F}$ as the Stackelberg leader (first mover) and $\mathrm{S}$ as the follower (second 90 mover), $\mathrm{S}$ as the Stakelberg leader (first mover) and $\mathrm{F}$ as the follower (second mover), or $\mathrm{F}$ and $\mathrm{S}$ 91 are equally powerful and, hence, move simultaneously (Choi 1991). Then, our allocation 92 problem is to determine who should be entrusted with the right of offering the wholesale price 93 contract to enforce social responsibility in the supply chain (hereafter, referred to as the 94 responsibility holder) under each of the three power structures. Depending on whether $\mathrm{F}$ or $\mathrm{S}$ is 95 the social responsibility holder and which power structure is considered, six scenarios may arise. 96 Game-theoretical analyses are first conducted to obtain the equilibriums for the six cases. The 97 allocation decisions are subsequently assessed based on both economic and CSR performance 98 criteria by employing the methodology of comparative institutional analysis that is widely 99 adopted in institutional economics literature (Coase 1960; Williamson 1985). For the economic 100 performance criterion, the system-wide profit is chosen as a proxy of efficiency to determine the 101 optimal allocation scheme, and this choice is consistent with the concept of strategic CSR (Baron 102 2001). For the CSR performance criterion, the optimal allocation decision is obtained by 103 maximizing the global CSR performance for the supply chain.

104 Our model is related to Gurnani et el. (2007), Xiao and Yang (2008) and Tsay and Agrawal 105 (2000) in the following two aspects. First, our CSR-sensitive demand is similar to the quality106 sensitive demand in Gurnani et el. (2007) and the service-sensitive demand in Xiao and Yang 107 (2008) and Tsay and Agrawal (2000). Second, for our CSR cost function, Xiao and Yang (2008) 108 and Tsay and Agrawal (2000) assume service cost functions in the same quadratic form that is 109 independent of selling quantity, while Gurnani et el. (2007) introduce a quality cost function with 110 both sales-irrelevant and sales-relevant components. In the aforesaid research, the authors focus 111 on equilibrium variables such as quality/service levels, prices, sales and profits, and it is not a 112 concern how different arrangements of quality/service pricing right affect the supply chain 113 system-wide profit (or efficiency). In this paper, we investigate both equilibrium variables (if 114 CSR were viewed as quality/service level) and the impact of different allocation schemes.

115 The rest of the article is organized as follows. Sections 2 and 3 present the basic model and 116 the corresponding equilibriums. Section 4 reports our main results, followed by some discussions

\footnotetext{
${ }^{3}$ We use wholesale price contracts because they are commonly observed in practice (Cachon 2003).

${ }^{4}$ For example, the Starbucks' sustainability conversion and performance price premiums (\$0.05 per pound) in its CAFÉ program demand a host of socially responsible practices (Lee et al. 2007).
} 
117 in Section 5. Finally, some concluding remarks are provided in section 6.

\section{The Model}

119 Consider a supply chain with two members, an upstream supplier S and a downstream firm

120 F. The global CSR performance of the supply chain is measured by a variable $y^{5}$. To achieve this

121 CSR performance level, certain investment has to be committed. Assume that this cost only 122 incurs by the supplier (but will be shared with the firm F via a wholesale price contract) and 123 takes a quadratic form ${ }^{6}: C(y)=c y^{2} / 2$, which is independent of the production quantity. In addition 124 to the social cost, a constant unit production cost $c_{0}$ is also incurred by S. The social 125 responsibility commitment by $\mathrm{S}$ is expected to be compensated by $\mathrm{F}$ through a wholesale price 126 contract that stipulates $\mathrm{F}$ to purchase product from $\mathrm{S}$ at a unit social-performance dependent 127 wholesale price $w(y)$ :

$$
w(y)=w_{0}+k y,
$$

129 where $w_{0}>0$ is a component that is independent of CSR performance, and $k \geq 0$ represents the marginal impact of CSR performance on the wholesale price.

Given a CSR performance level $y$, the larger the $k$ value, the more $\mathrm{F}$ is taking on the social responsibility for the supply chain. When $k$ is zero, all social responsibility for the supply chain will be solely assumed by S. On the other hand, when $k$ approaches infinity, all social 134 responsibility will be shifted to F. Therefore, it is reasonable to put a cap $\bar{k}$ on $k$ to make the contract implementable. It is obvious that the wholesale price in (1) serves as a mechanism to 136 share the social responsibility between $\mathrm{S}$ and $\mathrm{F}$ and $k$ plays a crucial role in achieving an 137 equitable transfer of social cost from S to F. Two key issues in allocating social responsibility 138 between the supply chain members $\mathrm{S}$ and $\mathrm{F}$ are who should be entrusted with the right of offering the wholesale price contract and what upper limit $\bar{k}$ should be placed on $k$.

F then sells the product in a consumer market characterized by a demand function

$$
p=A(y)-\frac{1}{2} b q
$$

142 where $p \geq 0$ and $q \geq 0$ are the price and the demand quantity, respectively, $b>0$ indicates the slope

\footnotetext{
${ }^{5}$ CSR performance can be measured by investment in CSR activities such as mitigating pollutant emission, improving working conditions, philanthropic donations.

${ }^{6}$ Röller (1990) theoretically shows that a quadratic cost function can behave well for analyzing global cost concepts (e.g. diminishing marginal returns) by properly choosing the parameters. In addition, quadratic cost functions are employed in many application studies (see, for example, Perry and Porter 1985, Rath and Zhao 2001, Kwoka 2002). Particularly, in the OM/OR area, Tsay and Agrawal (2000), Gurnani et el. (2007), Xiao and Yang (2008) also make this assumption for their cost function.
} 
143 of the demand curve, $A(y)$ characterizes the impact of CSR performance (denoted by $y \geq 0$ ) on

144 the final consumer market and is assumed to take the following form

$$
A(y)=a_{0}+a y,
$$

146 where $a_{0}>0$ captures the base willingness-to-pay from consumers, and $a>0$ stands for the 147 marginal impact of CSR performance on additional willingness-to-pay. This assumption is consistent with Mohr and Webb’s (2005) empirical results from a national sample that CSR has a positive impact on consumer purchase intent.

With these assumptions, the profit function for $\mathrm{F}$ is given as

Similarly, the profit for S is

$$
\Pi^{S}(q, y, k)=\left(w_{0}+k y\right) q-c_{0} q-\frac{1}{2} c y^{2} .
$$

Furthermore, we assume $w_{0}=c_{0}$ for the sake of analytical tractability ${ }^{7}$ and denote $A_{0}=a_{0}-w_{0}=a_{0}-c_{0}>0$ for notational simplification, then we have

$$
\begin{gathered}
\Pi^{F}(q, y, k)=\left(A_{0}+a y-k y\right) q-\frac{1}{2} b q^{2}, \\
\Pi^{S}(q, y, k)=k y q-\frac{1}{2} c y^{2} .
\end{gathered}
$$

The channel profit of the supply chain system is thus derived as

$$
\Pi^{T}(q, y)=\Pi^{F}(q, y, k)+\Pi^{S}(q, y, k)=\left(A_{0}+a y\right) q-\frac{1}{2} b q^{2}-\frac{1}{2} c y^{2} .
$$

Now F and S are treated as two economic agents. Following Choi (1991), the bargaining power in the supply chain is characterized by the Stackelberg leadership model. Three scenarios may arise: (1) Upstream Stackelberg (US) where S has more bargaining power than $\mathrm{F}$ and, hence, takes the first move; (2) Downstream Stackelberg (DS) where F has more bargaining power than $\mathrm{S}$ and, hence, moves first; and (3) Vertical Nash (VN) where S and F have equal bargaining power and, hence, move simultaneously.

According to the duality of rights and obligations (responsibilities), the responsibility holder

\footnotetext{
${ }^{7}$ The key motivation of assuming $w_{0}=c_{0}$ is to exclude the impact of production cost on the supplier's CSR decision so that we can isolate the supplier's CSR behavior and focus on examining how CSR commitments affect supply chain operations, and eventually analyze the impacts of different CSR allocation schemes on the efficiency of the whole supply chain (the system-wide profit).
} 
167 is assumed to have the right of offering a wholesale price contract $k \in[0, \bar{k}]$, where $\bar{k} \in(0, \infty)$

168 describes how strong the right corresponds to the social responsibility. Understandably, the 169 greater $\bar{k}$ is, the larger the margin of wholesale price contracts from which the responsibility 170 holder is allowed to choose, corresponding to a stronger right for the responsibility holder. Given 171 this interpretation, if the social responsibility is allocated to F, it will offer to S a wholesale price 172 by selecting $k \in[0, \bar{k}]$ and also order $q$ units such that its profit $\Pi^{F}$ is maximized and $\mathrm{S}$, in this 173 case, will choose $y$ to maximize its own profit $\Pi^{s}$; on the other hand, if $\mathrm{S}$ is allocated as the 174 social responsibility holder, it will offer to $\mathrm{F}$ a contract characterized by $k$ and determine a CSR 175 performance level $y$ to maximize $\Pi^{s}$ and $\mathrm{F}$ will thus select $q$ to maximize $\Pi^{F}$. Therefore, the 176 allocation of social responsibility is twofold: who is the responsibility holder to offer $k$ and what 177 cap $\bar{k}$ is placed on $k$. This allocation decision can thus be depicted by $(X, \bar{k}) \in\{F, S\} \times(0, \infty)$. As for 178 the timing of the $k$ decision, the base model in Sections 3 and 4 assumes that it is made at the 179 same time as the other decision variable controlled by the responsibility holder. Section 5, on the 180 other hand, examines the situation that $k$ is offered by the responsibility holder prior to the other 181 two decision variables $q$ and $y$ are determined by $\mathrm{F}$ and S, respectively.

182 Finally, by combining the choice of a responsibility holder (F or S) and a power structure 183 (US, DS, or VN), six scenarios arise and are hereafter labeled as S-US, S-DS, S-VN, F-US, F-DS, 184 and F-VN games, respectively, where the first letter indicates the responsibility holder and the 185 last two letters identify the power structure. For instance, in the S-US game, the supplier S is the 186 responsibility holder and the Stackelberg leader and, hence, $\mathrm{S}$ is entitled to choose $k$ as a 187 responsibility holder and determines its variable $y$ first as a Stackelberg leader, subsequently, F 188 as the Stackelberg follower responds with $q$ to the choices by S. The other five labels can be 189 interpreted in a similar fashion. Next, the six games are examined and their equilibriums are 190 obtained.

\section{Equilibriums}

192 To make the following analysis meaningful, assume $a^{2}<b c$ to guarantee the system-wide optimal 193 profit and CSR performance for the supply chain to be greater than zero.

\section{The integrative case}

195 First the integrative case is considered with social responsibility. The first-order conditions 


$$
\left(A_{0}+a y\right)-b q=0
$$

$$
a q-c y=0 .
$$

Solving these two equations simultaneously yields

$$
y_{I}^{*}=\frac{A_{0} a}{b c-a^{2}} ; q_{I}^{*}=\frac{A_{0} c}{b c-a^{2}} .
$$

The maximum profit of the supply chain system is

$$
\Pi_{I}^{*}=\frac{A_{0}^{2} c}{2\left(b c-a^{2}\right)} .
$$

When social responsibility is not considered in the model with all terms associated with $y$ 204 being removed, the optimal quantity and system-wide profit can be conveniently obtained as 205 follows

$$
q_{N}^{*}=\frac{A_{0}}{b} ; \Pi_{N}^{*}=\frac{A_{0}^{2}}{2 b} .
$$

\section{The S-US game}

In the S-US game, the supplier S offers the wholesale price contract $(k)$ and chooses $y$,

210 function for $\mathrm{F}$ is

$$
q(y, k)=\frac{A_{0}+(a-k) y}{b} .
$$

Then the supplier's profit function can be rewritten as

$$
\Pi_{S-U S}^{S}(q(y, k), y, k)=\frac{A_{0} k y+(a-k) k y^{2}}{b}-\frac{1}{2} c y^{2} .
$$

214 Clearly, this profit function is concave both in $y$ for a given $k$ and in $k$ for a given $y$, 215 thereby validating Zabel's (1970) method of first optimizing $y$ for a given $k$ and searching over

216 the resulting optimal trajectory to find the optimal $k$. The first-order condition with respect to $y$ 217 is

$$
\frac{\partial \Pi_{S-U S}^{S}}{\partial y}=\frac{A_{0} k+2(a-k) k y}{b}-c y=0 \Rightarrow y(k)=\frac{A_{0} k}{b c-2 k(a-k)} .
$$

\footnotetext{
${ }^{8}$ The second order condition is easy to check. For remaining discussions, all second order conditions can be checked in a straightforward manner and, hence, are omitted in the article.
} 
Note that $b c-2 k(a-k)=b c-2 k a+2 k^{2} \geq b c-2 k a+k^{2}=(a-k)^{2}+b c-a^{2}$. Due to the aforesaid assumption $a^{2}<b c$, it is confirmed that the denominator is positive. Therefore, the first-order

223 derivative is positive, or equivalently, $\Pi_{s-U S}^{s}$ increases in $k$, for all $k<b c / a$, and $\Pi_{s-U s}^{s}$ decreases in

$224 k$ for all $k>b c / a$. This indicates that $\Pi_{s-U s}^{s}$ is unimodal in $k$. So, if $k$ is capped before $\Pi_{s-U s}^{s}$ reaches 225 its maximum at $k=b c / a$, i.e., for all $\bar{k} \leq b c / a$, the optimal wholesale price contract will occur at 226 the boundary, $k_{s-u s}^{*}=\bar{k}$. Otherwise, if the cap for $k$ is extended beyond $k=b c / a$, i.e., for all $\bar{k}>b c / a$, $227 k_{s-U s}^{*}=b c / a$. Plugging the optimal $k_{s-U s}^{*}$ into (9), (7), (4), (5) and (6), we can calculate the 228 equilibrium CSR performance $\left(y_{s-U S}^{*}\right)$ and product quantity $\left(q_{s-U S}^{*}\right)$, as well as the equilibrium 229 profits for the firm, the supplier, and the supply chain system. These equilibrium variables are 230 summarized in Proposition 1.

231 Proposition 1: The subgame perfect equilibrium of the S-US game is summarized as

232 (i) If $\bar{k} \leq b c / a$, the equilibrium variables are

$$
\begin{gathered}
k_{S-U S}^{*}=\bar{k} ; y_{S-U S}^{*}=\frac{A_{0} \bar{k}}{b c-2 \bar{k}(a-\bar{k})} ; q_{S-U S}^{*}=\frac{A_{0}[b c-\bar{k}(a-\bar{k})]}{b[b c-2 \bar{k}(a-\bar{k})]} ; \\
\Pi_{S-U S}^{S^{*}}=\frac{A_{0}^{2} \bar{k}^{2}}{2 b[b c-2 \bar{k}(a-\bar{k})]} ; \Pi_{S-U S}^{F^{*}}=\frac{A_{0}^{2}[b c-\bar{k}(a-\bar{k})]^{2}}{2 b[b c-2 \bar{k}(a-\bar{k})]^{2}} ; \Pi_{S-U S}^{T^{*}}=\frac{A_{0}^{2}}{2 b}\left(\frac{\bar{k}^{2}}{b c-2 \bar{k}(a-\bar{k})}+\frac{[b c-\bar{k}(a-\bar{k})]^{2}}{[b c-2 \bar{k}(a-\bar{k})]^{2}} .\right.
\end{gathered}
$$

(ii) If $\bar{k}>b c / a$, the equilibrium variables are

$$
\begin{gathered}
k_{S-U S}^{*}=b c / a ; y_{S-U S}^{*}=\frac{A_{0} a}{2 b c-a^{2}} ; q_{S-U S}^{*}=\frac{A_{0} c}{2 b c-a^{2}} ; \\
\Pi_{S-U S}^{S^{*}}=\frac{A_{0}^{2} c}{2\left(2 b c-a^{2}\right)} ; \Pi_{S-U S}^{F^{*}}=\frac{A_{0}^{2} b c^{2}}{2\left(2 b c-a^{2}\right)^{2}} ; \Pi_{S-U S}^{T^{*}}=\frac{A_{0}^{2} c\left(3 b c-a^{2}\right)}{2\left(2 b c-a^{2}\right)^{2}} .
\end{gathered}
$$

\section{The F-US game}

The F-US game is similar to the S-US case except that F rather than S offers the wholesale price contract characterized by $k$. Given $y$ from S, F determines $k$ and $q$ to maximize

242 condition with respect to $q$ immediately implies that, for any $k \in[0, \bar{k}], \mathrm{F}$ orders $q=A_{0} / b$ with a

243 maximal profit $\Pi_{F-u s}^{F}(q, y, k)=\Pi^{F}\left(A_{0} / b, 0, k\right)=A_{0}^{2} /(2 b)$. As $y=0$ in this case, $k$ becomes irrelevant. 244 Therefore, although $k$ may assume any value between 0 and $\bar{k}$, for convenience, we set it at 
$k(y)=0$ to break ties. On the other hand, if S commits to $y>0$, for any $q>0, \Pi_{F-U S}^{F}(q, y, k)$ strictly decreases in $k$, then the optimal wholesale price contract is $k=0$, and the corresponding optimal order is given as $q=\left(A_{0}+a y\right) / b$. In this case, $\Pi_{F-U S}^{F}(q, y, k)=\Pi^{F}\left(\left(A_{0}+a y\right) / b, y, 0\right)=\left(A_{0}+a y\right)^{2} /(2 b)>0$. In addition, $y>0$ and $q=0$ together imply that $\Pi_{F-U S}^{F}(q, y, k)=\Pi^{F}(0, y, k)=0$ for any $k \geq 0$. Thus, the optimal reaction to $y>0$ can be expressed as $q=\left(A_{0}+a y\right) / b$ and $k=0$. In summary, the optimal 250 reaction from $\mathrm{F}$ is

$$
(q(y), k(y))=\left(\frac{A_{0}+a y}{b}, 0\right) .
$$

By backward induction, the profit function for $\mathrm{S}$ is rewritten as $\Pi_{F-U s}^{S}(y) \equiv \Pi^{s}(q(y), y, k(y))$, where $q(y)$ and $k(y)$ are given in (10). If S chooses $y=0$, then its profit becomes $\Pi_{F-u s}^{S}(y)=\Pi^{S}\left(A_{0} / b, 0,0\right)=0$.

254 But if it selects $y>0$, its profit is $\Pi_{F-U S}^{S}(y)=\Pi^{s}\left(\left(A_{0}+a y\right) / b, y, 0\right)=-c y^{2} / 2<0$. Thus, the optimal decision for $\mathrm{S}$ is $y_{F-U S}^{*}=0$. Therefore, the subgame perfect equilibrium of the F-US game can be 256 obtained as shown in Proposition 2.

257 Proposition 2: The subgame perfect equilibrium of the F-US game can be summarized as

$$
\begin{gathered}
k_{F-U S}^{*}=0 ; y_{F-U S}^{*}=0 ; q_{F-U S}^{*}=\frac{A_{0}}{b} ; \\
\Pi_{F-U S}^{S^{*}}=0 ; \Pi_{F-U S}^{F^{*}}=\frac{A_{0}^{2}}{2 b} ; \Pi_{F-U S}^{T *}=\frac{A_{0}^{2}}{2 b} .
\end{gathered}
$$

\section{The S-DS game}

In the S-DS game, $\mathrm{F}$ chooses $q$ first and, then given $q$, S reacts with $k$ and $y$ to maximize $\Pi_{s-D S}^{s}(q, y, k) \equiv \Pi^{s}(q, y, k)$. Once again, backward induction is employed to obtain its equilibrium. First, if $\mathrm{F}$ orders $q=0$, it becomes trivial with $y=0$ and $k=k^{\prime}$ by $\mathrm{S}$, where $k^{\prime}$ is a real number arbitrarily picked from $k \in[0, \bar{k}]$. On the other hand, if $q>0$, the optimal reaction $(k, y)$ by S must satisfy the first-order condition with respect to $y$, i.e. $k q=c y$. Notice that $k=0$ implies $y=0$, 266 thereby $\Pi_{S-D S}^{s}(q, 0,0)=0$. Note further that $k>0$ means $y>0$, and it follows that $267 \partial \prod_{s-D S}^{S}(q, y, k) / \partial k=y q>0$, indicating that the profit for S strictly increases in $k$ and, hence, reaches its maximum at $\bar{k}$. In addition, for all $(k, y)>0$ with $k q=c y, \Pi_{s-D S}^{s}(q, y, k)=(k q)^{2} /(2 c)>0$. Therefore, the optimal reaction $(k, y)$ to $q>0$ is $(\bar{k}, \bar{k} q / c)$. To summarize, the reaction function from $\mathrm{S}$ is expressed as 


$$
(k(q), y(q))=\left\{\begin{array}{ll}
\left(k^{\prime}, 0\right), & \text { if } q=0 \\
\left(\bar{k}, \frac{\bar{k} q}{c}\right), & \text { if } q>0
\end{array} .\right.
$$

Given (11), if the firm chooses $q=0$, its profit is $\Pi_{S-D S}^{F}(q) \equiv \Pi^{F}(q, y(q), k(q))=0$. For $q>0$, its 273 profit function can be rewritten as

$$
\Pi_{S-D S}^{F}(q)=\Pi^{F}(q, y(q), k(q))=A_{0} q+\left(\frac{\bar{k}(a-\bar{k})}{c}-\frac{b}{2}\right) q^{2} .
$$

275

concave. The first-order condition with respect to $q$ immediately yields

$$
q_{S-D S}^{*}=\frac{A_{0} c}{b c-2 \bar{k}(a-\bar{k})} \text {. }
$$

Finally, given that $q_{S-D S}^{*}>0$ as its denominator and numerator are positive, the optimal response $(k(q), y(q))$ from S can be easily obtained from (11). Plugging them into (4), (5) and (6), one can determine the equilibrium profit for S, F, and the supply chain system. All equilibrium variables of the S-DS game can thus be furnished as Proposition 3 below.

282 Proposition 3: The subgame perfect equilibrium of the S-DS game can be summarized as

$$
\begin{gathered}
k_{S-D S}^{*}=\bar{k} ; y_{S-D S}^{*}=\frac{A_{0} \bar{k}}{b c-2 \bar{k}(a-\bar{k})} ; q_{S-D S}^{*}=\frac{A_{0} c}{b c-2 \bar{k}(a-\bar{k})} ; \\
\Pi_{S-D S}^{S^{*}}=\frac{A_{0}^{2} c \bar{k}^{2}}{2[b c-2 \bar{k}(a-\bar{k})]^{2}} ; \Pi_{S-D S}^{F^{*}}=\frac{A_{0}^{2} c}{2[b c-2 \bar{k}(a-\bar{k})]} ; \Pi_{S-D S}^{T^{*}}=\frac{A_{0}^{2} c\left(b c-2 \bar{k} a+3 \bar{k}^{2}\right)}{2[b c-2 \bar{k}(a-\bar{k})]^{2}} .
\end{gathered}
$$

\section{The F-DS game}

In the F-DS game, $\mathrm{F}$ chooses $k$ and $q$ first, followed by $\mathrm{S}$ selecting $y$ to maximize $\Pi_{F-D S}^{S}(q, y, k) \equiv \Pi^{S}(q, y, k)$ under the given $k$ and $q$. The reaction function for $\mathrm{S}$ is thus

$$
y(k, q)=\frac{k q}{c} .
$$

Given (12), the profit function for $\mathrm{F}$ is

$$
\Pi_{F-D S}^{F}(q, k) \equiv \Pi^{F}(q, y(k, q), k)=A_{0} q+\left(\frac{k(a-k)}{c}-\frac{b}{2}\right) q^{2} .
$$

This profit function is concave both in $q$ for a given $k$ and in $k$ for a given $q>0$, 292 permitting the application of Zabel's (1970) method for optimization. Next, we first optimize $q$ 293 for a given $k$ and, then find the optimal $k$. From the first-order condition with respect to $q$, we 294 have 


$$
q(k)=\frac{A_{0} c}{b c-2 k(a-k)} .
$$

Substituting (14) into (13) and taking the derivative with respect to $k$ yield

$$
\frac{\partial \Pi_{F-D S}^{F}}{\partial k}=\frac{(a-2 k)[q(k)]^{2}}{c} .
$$

This indicates that $\Pi_{F-D S}^{F}$ increases in $k$ for $k<a / 2$ and decreases in $k$ if $k>a / 2$ and, hence, $299 \Pi_{F-D S}^{F}$ is unimodal. Thus, $k_{F-D S}^{*}=\bar{k}$ if $\bar{k}<a / 2$, otherwise $k_{F-D S}^{*}=a / 2$. By (14), (12), (4), (5) and (6), 300 we can determine the equilibrium variables as shown in Proposition 4.

301 Proposition 4: The subgame perfect equilibrium of the F-DS game can be summarized as

(ii) If $\bar{k}>a / 2$, the equilibrium variables are

\section{The S-VN game}

Under the assumption of the S-VN game, S and F determine their variables simultaneously, where S furnishes $k$ and $y$ and F provides a quantity $q$. It is easy to verify that (7) and (11) are the reaction functions for $\mathrm{F}$ and $\mathrm{S}$, respectively. If $q=0,(11)$ implies that $y=0$ and $k$ is arbitrary, but (7) indicates that $q(0, k)=A_{0} / b>0$. This means that $q$ cannot be zero in the equilibrium. For

312 it for $q$, one can have

$$
\Pi_{F-D S}^{S^{*}}=\frac{A_{0}^{2} a^{2} c}{2\left(2 b c-a^{2}\right)^{2}} ; \Pi_{F-D S}^{F^{*}}=\frac{A_{0}^{2} c}{2 b c-a^{2}} ; \Pi_{F-D S}^{T^{*}}=\frac{A_{0}^{2} c\left(4 b c-a^{2}\right)}{2\left(2 b c-a^{2}\right)^{2}} .
$$
$q>0$, (11) implies that $k=\bar{k}$ and $y=\bar{k} q / c$. Substituting these two equations into (7) and solving

With this result, it is straightforward to derive other variables in the equilibrium as given in the following proposition.

$$
q_{S-V N}^{*}=\frac{A_{0} c}{b c-\bar{k}(a-\bar{k})} .
$$

Proposition 5: The Nash equilibrium of the S-VN game can be summarized as

$$
k_{S-V N}^{*}=\bar{k} ; y_{S-V N}^{*}=\frac{A_{0} \bar{k}}{b c-\bar{k}(a-\bar{k})} ; q_{S-V N}^{*}=\frac{A_{0} c}{b c-\bar{k}(a-\bar{k})} ;
$$

$$
\Pi_{S-V N}^{S^{*}}=\frac{A_{0}^{2} c \bar{k}^{2}}{2[b c-\bar{k}(a-\bar{k})]^{2}} ; \Pi_{S-V N}^{F^{*}}=\frac{A_{0}^{2} c}{2[b c-\bar{k}(a-\bar{k})]} ; \Pi_{S-V N}^{T^{*}}=\frac{A_{0}^{2} c\left(b c-\bar{k} a+2 \bar{k}^{2}\right)}{2[b c-\bar{k}(a-\bar{k})]^{2}} .
$$


In the F-VN game, F determines $k$ and $q$ at the same time as S gives $y$. Clearly, (10) and (12) are the reaction functions for $F$ and S, respectively. (10) and (12) are next solved 322 simultaneously. If $y=0$, we have $q=A_{0} / b$ from (10), and (12) further implies $k=0$. Thus, $323(k, q, y)=\left(0, A_{0} / b, 0\right)$ is a Nash equilibrium for the F-VN game. It is actually the unique Nash equilibrium. As a matter of fact, if $y>0$, (10) implies that $k=0$ and $q=\left(A_{0}+a y\right) / b>0$, contradictory to (12). Therefore, $(k, q, y)=\left(0, A_{0} / b, 0\right)$ is the unique triplet that satisfies both (10) and (12) simultaneously, leading to the following proposition.

Proposition 6: The Nash equilibrium of the F-VN game can be summarized as

Remark: Propositions 1-6 demonstrate that the power structure has a significant impact on the behavior of the responsibility holder. If a supply chain member is entrusted as a responsibility holder who offers the wholesale price contract characterized by $k$, it seems to behave in an equitable manner only if it assumes the leadership position. On the one hand, if responsibility holder S is the Stackelberg leader, corresponding to the S-US case, it will always share social responsibility with $\mathrm{F}$ at an optimal level of $k_{S-U S}^{*}=b c / a$ if $\bar{k}>b c / a$ or $k_{S-U S}^{*}=\bar{k}$ if $\bar{k}<b c / a$. Similarly, if responsibility holder F is the Stackelberg leader in the F-DS case, F will offer $k_{F-D S}^{*}=a / 2$ to take its share in achieving the equilibrium CSR performance level. On the other hand, if social responsibility of the supply chain is allocated to $\mathrm{S}$, but it is not the Stackelberg leader in the S-DS or S-VN case, $\mathrm{S}$ will always push the $k$ value to its maximum, i.e., $k_{S-D S}^{*}=\bar{k}$ or $k_{S-V N}^{*}=\bar{k}$. If there is no restriction on $\bar{k}$, i.e., $\bar{k} \rightarrow \infty$, S will not pull its weight but transfer all of its social responsibility investment to $\mathrm{F}$ via the wholesale price contract. This observation indicates that the right of offering $k$ for $S$ should come with a restriction on the upper limit of $k$, which may be imposed by a third party, for instance, a government agency, or through a negotiation between the supply chain partners so that social responsibility is indeed equitably shared. In a similar

345 fashion, one can examine the cases that $\mathrm{F}$ is the responsibility holder but not the Stackelberg 346 leader in the F-US or F-VN games. In both cases, F sets $k^{*}=0$ and refuses to share any CSR investment with S, eventually leading to no CSR performance for the supply chain $\left(y_{F-U S}^{*}=0\right.$ and 
$\left.y_{F-V N}^{*}=0\right)$. This result shows the other side of the coin: when $\mathrm{F}$ is entrusted as the responsibility

holder to determine the wholesale price, a lower bound should be placed on $k$ to ensure a reasonable transfer of social responsibility cost from S so that the undesirable case of zero CSR

351 performance is avoided for the supply chain. Once again, this lower limit could be imposed by a 352 third party or negotiated between $\mathrm{F}$ and S.

353 In summary, the equilibrium value of the parameter $k$ characterizes how CSR investment is 354 expected to be shared between $\mathrm{S}$ and $\mathrm{F}$, and the CSR investment tends to be shared in an 355 equitable manner if the Stackelberg leader is allocated to decide $k$. Intuitively, in the US and DS 356 cases, the leader's profit depends on the follower's response (or threat) and the leader is thus able 357 to take advantage of its leadership position to stimulate (or guide) the follower by choosing a 358 reasonable $k$ to equitably share the CSR investment. On the contrary, if the follower is entrusted 359 with the right of selecting $k$, it knows that its decision on $k$ will be final as the leader has 360 already committed to its actions. As such, the follower does not have any economic incentive to 361 pull its weight. In the VN case, neither stimulation nor threat is possible because $\mathrm{S}$ and $\mathrm{F}$ have to 362 move simultaneously without any prior knowledge of commitments from their partner. Therefore, 363 each party with the right of determining $k$, in its best economic interests, pushes $k$ towards its 364 boundary ( $\bar{k}$ in Proposition 5 and 0 in Proposition 6), thereby forcing its partner to take on as 365 much CSR investment cost as possible.

3664 Main results

367 This section analyzes the equilibriums and derives the optimal allocation of social responsibility 368 according to the methodology of comparative institutional analysis. Implications on business 369 practice are also explored for the resulting social responsibility allocation scheme within a 370 supply chain management context.

\section{$371 \quad 4.1$ Optimal responsibility allocations based on the economic performance criterion}

372 In Section 3, equilibriums are obtained by examining each supply chain member's strategic 373 behavior under each of the six aforesaid scenarios. In equilibrium, each member has chosen its 374 optimal strategy to maximize its own profit. Here, we shall employ the comparative institutional 375 analysis approach to investigate the equilibriums and determine the optimal social responsibility 376 allocation scheme that maximizes the total equilibrium profit for the channel under each of the 
three power structures ${ }^{9}$.

The US case

From Proposition 1, we have

$$
\Pi_{S-U S}^{T^{*}}(\bar{k})=\left\{\begin{array}{ll}
\frac{A_{0}^{2}}{2 b}\left(\frac{\bar{k}^{2}}{b c-2 \bar{k}(a-\bar{k})}+\frac{[b c-\bar{k}(a-\bar{k})]^{2}}{[b c-2 \bar{k}(a-\bar{k})]^{2}}\right), & \text { if } \bar{k} \leq \frac{b c}{a} \\
\frac{A_{0}^{2} c\left(3 b c-a^{2}\right)}{2\left(2 b c-a^{2}\right)^{2}}, & \text { if } \bar{k}>\frac{b c}{a}
\end{array} .\right.
$$

For all $\bar{k} \in(0, b c / a]$, we have

where $F(\bar{k})=-2 a \bar{k}^{4}+2 a^{2} \bar{k}^{3}-b c\left(a^{2}+b c\right) \bar{k}+a b^{2} c^{2}$.

Then $F^{\prime}(\bar{k})=-8 a \bar{k}^{3}+6 a^{2} \bar{k}^{2}-b c\left(a^{2}+b c\right)$ and $F^{\prime \prime}(\bar{k})=12 a \bar{k}(a-2 \bar{k})$. It follows that $F^{\prime}(\bar{k})$ increases for $\bar{k} \in(0, a / 2]$ and decreases for $\bar{k} \in(a / 2, \infty)$. Thus $F^{\prime}(\bar{k})$ is unimodal in $\bar{k}$ and attains its maximum at $\bar{k}=a / 2$ over $(0, \infty)$. Note that $F^{\prime}(a / 2)=a^{4} / 2-b c\left(a^{2}+b c\right)<0$ due to the assumption $a^{2}<b c$, therefore, $F^{\prime}(\bar{k})<0$ for all $\bar{k} \in(0, \infty)$. Further, as $F(0)=a b^{2} c^{2}>0$ and $F(a)=-a^{3} b c<0$, it implies that there exists a unique $\bar{k}_{e}^{*} \in(0, a) \subset(0, b c / a)$ such that $F\left(\bar{k}_{e}^{*}\right)=0, F(\bar{k})>0$ for $\bar{k}<\bar{k}_{e}^{*}$ and $F(\bar{k})<0$ for $\bar{k}>\bar{k}_{e}^{*}$. Thus (15) implies that $\mathrm{d} \Pi_{s-U s}^{T^{*}} / \mathrm{d} \bar{k}=0$ at $\bar{k}=\bar{k}_{e}^{*}$, $\mathrm{d} \Pi_{s-U s}^{T^{*}} / \mathrm{d} \bar{k}>0$ for $\bar{k}<\bar{k}_{e}^{*}$ and $\mathrm{d} \Pi_{s-U s}^{T^{*}} / \mathrm{d} \bar{k}<0$ for $\bar{k}>\bar{k}_{e}^{*}$. Therefore, $\Pi_{s-U s}^{T^{*}}$ is unimodal in $\bar{k}$ and attains its maximum at $\bar{k}=\bar{k}_{e}^{*}$ over $(0, \infty)$. profit for the F-US game, hence,

$$
\Pi_{F-U S}^{T^{*}}(\bar{k})=\frac{A_{0}^{2}}{2 b}<\frac{A_{0}^{2}}{2 b}\left(\frac{a^{2}}{b c}+1\right)=\Pi_{S-U S}^{T^{*}}(a)<\Pi_{S-U S}^{T^{*}}\left(\bar{k}_{e}^{*}\right) \text {, for any } \bar{k} \in(0, \infty) .
$$

$395 \mathrm{~S}$ will be allocated as the responsibility holder with the right to choose $k=\bar{k}_{e}^{*} \in\left[0, \bar{k}_{e}^{*}\right]$ when S is the 396 upstream Stackelberg leader.

\section{The DS case}

\section{From Proposition 3, we have}

${ }^{9}$ The comparative institutional analysis (Williamson 1985) suggests that transaction cost savings (efficiency enhancement) via actors' rational behavior drive the evolution of institutions. As such, comparative efficiency advantages dominate the choice of institutions. Applying this idea to our study, to determine who should be allocated the right of offering a wholesale price contract, a rational recommendation is the one who is able to achieve higher system-wide profit for the supply chain. 


$$
\frac{\mathrm{d} \Pi_{S-D S}^{T^{*}}}{\mathrm{~d} \bar{k}}=\frac{A_{0}^{2} c\left(-6 \bar{k}^{3}+6 a \bar{k}^{2}-\left(2 a^{2}+b c\right) \bar{k}+a b c\right)}{[b c-2 \bar{k}(a-\bar{k})]^{3}}=\frac{A_{0}^{2} c G(\bar{k})}{[b c-2 \bar{k}(a-\bar{k})]^{3}},
$$

400

401

402

where $G(\bar{k})=-6 \bar{k}^{3}+6 a \bar{k}^{2}-\left(2 a^{2}+b c\right) \bar{k}+a b c$.

As $G^{\prime}(\bar{k})=-18 \bar{k}^{2}+12 a \bar{k}-\left(2 a^{2}+b c\right)=-2(3 \bar{k}-a)^{2}-b c<0, G^{\prime}(\bar{k})<0$ for all $\bar{k} \in(0, \infty)$, implying that $G(\bar{k})$ decreases in $\bar{k}$ for $\bar{k} \in(0, \infty)$. Note that $G\left(\frac{a}{2}\right)=a\left(\frac{b c}{2}-\frac{a^{2}}{4}\right)=\frac{a}{4}\left(2 b c-a^{2}\right)>0$ and $G(a)=-2 a^{2}<0$, there exists a unique $\bar{k}_{e}^{* *} \in\left(\frac{a}{2}, a\right)$ such that $G\left(\bar{k}_{e}^{* *}\right)=0, G(\bar{k})>0$ for $\bar{k}<\bar{k}_{e}^{* *}$ and $G(\bar{k})<0$ for $\bar{k}>\bar{k}_{e}^{* *}$. It follows from (16) that $\mathrm{d} \Pi_{S-D S}^{T^{* *}} / \mathrm{d} \bar{k}=0$ at $\bar{k}=\bar{k}_{e}^{* *}, \mathrm{~d} \Pi_{S-D S}^{T^{*}} / \mathrm{d} \bar{k}>0$ for $\bar{k}<\bar{k}_{e}^{* *}$ and $\mathrm{d} \Pi_{S-D S}^{T^{*}} / \mathrm{d} \bar{k}<0$ for $\bar{k}>\bar{k}_{e}^{* *}$. Thus $\Pi_{S-D S}^{T^{*}}$ is unimodal in $\bar{k}$ and attains its maximum at $\bar{k}=\bar{k}_{e}^{* *}$.

From Proposition 4, we have

$$
\Pi_{F-D S}^{T^{*}}(\bar{k})=\left\{\begin{array}{ll}
\Pi_{S-D S}^{T^{*}}(\bar{k}), & \text { if } \bar{k} \leq \frac{a}{2} \\
\frac{A_{0}^{2} c\left(4 b c-a^{2}\right)}{2\left(2 b c-a^{2}\right)^{2}}, & \text { if } \bar{k}>\frac{a}{2}
\end{array} .\right.
$$

It is confirmed that $\Pi_{F-D S}^{T^{*}}(\bar{k})$ is continuous in $\bar{k}$. Given that $\Pi_{S-D S}^{T^{*}}(\bar{k})$ increases in $\bar{k}$ when $\bar{k}<\frac{a}{2}$, (17) indicates that $\Pi_{F-D S}^{T^{*}}(\bar{k})$ reaches its maximum at $\bar{k}=\frac{a}{2}$. Therefore, for any $\bar{k} \in(0, \infty)$

$$
\Pi_{F-D S}^{T^{*}}(\bar{k}) \leq \Pi_{F-D S}^{T^{*}}\left(\frac{a}{2}\right)=\frac{A_{0}^{2} c\left(4 b c-a^{2}\right)}{2\left(2 b c-a^{2}\right)^{2}}=\Pi_{S-D S}^{T^{*}}\left(\frac{a}{2}\right)<\Pi_{S-D S}^{T^{*}}\left(\bar{k}_{e}^{* *}\right) .
$$

Thus $\left(S, \bar{k}_{e}^{* *}\right)$ arises as the unique optimal responsibility allocation in the DS case.

\section{The VN case}

From Proposition 5, for $\bar{k} \in(0, \infty)$, we have

$$
\frac{\mathrm{d} \Pi_{s-V N}^{T^{*}}}{\mathrm{~d} \bar{k}}=\frac{A_{0}^{2} c\left(-4 \bar{k}^{3}+3 a \bar{k}^{2}-a^{2} \bar{k}+a b c\right)}{2[b c-\bar{k}(a-\bar{k})]^{3}}=\frac{A_{0}^{2} c H(\bar{k})}{2[b c-\bar{k}(a-\bar{k})]^{3}},
$$

where $H(\bar{k})=-4 \bar{k}^{3}+3 a \bar{k}^{2}-a^{2} \bar{k}+a b c$.

Then $H^{\prime}(\bar{k})=-12 \bar{k}^{2}+6 a \bar{k}-a^{2}=-3 \bar{k}^{2}-(3 \bar{k}-a)^{2}<0$, indicating that $H(\bar{k})$ decreases in $\bar{k}$. As $H(a / 2)=a\left(b c-a^{2} / 4\right)>0$ and $H(\sqrt{b c})=b c(a-\sqrt{b c})-a^{2} \sqrt{b c}<0$ (due to $a^{2}<b c$ ), there exists a unique $\bar{k}_{e}^{*+*+*} \in(a / 2, \sqrt{b c})$ such that $H\left(\bar{k}_{e}^{+*+*}\right)=0, H(\bar{k})>0$ for $\bar{k}<\bar{k}_{e}^{+*+*}$ and $H(\bar{k})<0$ for $\bar{k}>\bar{k}_{e}^{+*+*}$. Given that the denominator of (18) is positive, it follows that $\mathrm{d} \Pi_{s-V N}^{T^{*}} / \mathrm{d} \bar{k}=0$ at $\bar{k}=\bar{k}_{e}^{* * * *}, \mathrm{~d} \Pi_{s-V N}^{T_{N}^{*}} / \mathrm{d} \bar{k}>0$ for $\bar{k}<\bar{k}_{e}^{*+*}$ and $\mathrm{d} \Pi_{S-V N}^{T^{*}} / \mathrm{d} \bar{k}<0$ for $\bar{k}>\bar{k}_{e}^{* * *}$. Therefore, $\Pi_{S-V N}^{T^{*}}$ is unimodal in $\bar{k}$ and achieves its maximum at $\bar{k}=\bar{k}_{e}^{* * *}$ over $(0, \infty)$.

Furthermore, Proposition 6 indicates that a constant equilibrium channel profit is always attained for any $\bar{k}$ when $\mathrm{F}$ is responsible for determining $k \in[0, \bar{k}]$ and, hence, 


$$
\Pi_{F=V N}^{T^{*}}(\bar{k})=\frac{A_{0}^{2}}{2 b}<\frac{A_{0}^{2}\left(b c+a^{2}\right)}{2 b^{2} c}=\Pi_{S=V N}^{T^{*}}(a) \leq \Pi_{S=V N}^{T^{*}}\left(k_{e}^{* * *}\right) .
$$

Therefore $\left(S, \bar{k}_{e}^{* * *}\right)$ is the unique optimal responsibility allocation in the VN case.

These results can now be summarized as Proposition 7.

Proposition 7: According to the economic performance criterion, $\left(S, \bar{k}_{e}^{*}\right),\left(S, \bar{k}_{e}^{* * *}\right)$ and $\left(S, \bar{k}_{e}^{*+* *}\right)$ are the unique optimal responsibility allocation for the US, DS and VN cases, respectively.

Proposition 7 furnishes the optimal allocation schemes as well as the corresponding $\bar{k}$ values at optimality under the three power structures. Next, Corollaries 1-3 further establish that it remains optimal to entrust $\mathrm{S}$ with the right of offering the wholesale contract over a certain range of $\bar{k}$ values, even if they are not set at their corresponding optimality.

Corollary 1: If $b c \leq(3+\sqrt{5}) a^{2} / 2$, then $\Pi_{s-U S}^{T^{*}}(\bar{k}) \geq \Pi_{F-U S}^{T^{*}}(\bar{k})$ for $\bar{k} \in(0, \infty)$; otherwise, if $b c>(3+\sqrt{5}) a^{2} / 2$, there exists a unique $\bar{k}_{e}^{\#}>\bar{k}_{e}^{*}$ such that $\Pi_{S-U S}^{T^{*}}(\bar{k}) \geq \Pi_{F-U S}^{T^{*}}(\bar{k})$ for $\bar{k} \in\left(0, \bar{k}_{e}^{\#}\right]$ and $\Pi_{S-U S}^{T^{*}}(\bar{k})<\Pi_{F-U S}^{T^{*}}(\bar{k})$ for $\bar{k} \in\left(\bar{k}_{e}^{\#}, \infty\right)$.

Proof: Note that for all $\bar{k} \in(0, \infty), \Pi_{F-U S}^{T^{*}}(\bar{k})=A_{0}^{2} /(2 b)$ and $\lim _{\bar{k} \rightarrow 0} \Pi_{S-U S}^{T^{*}}(\bar{k})=A_{0}^{2} /(2 b)$. Earlier arguments indicate that $\Pi_{S-U S}^{T^{*}}(\bar{k})$ increases in $\bar{k}$ for $\bar{k} \in\left(0, \bar{k}_{e}^{*}\right]$ and decreases in $\bar{k}$ for $\bar{k} \in\left(\bar{k}_{e}^{*}, b c / a\right]$ and, then, stays constant at $\frac{A_{0}^{2} c\left(3 b c-a^{2}\right)}{2\left(2 b c-a^{2}\right)^{2}}$ for $\bar{k}>b c / a$. One can verify that

$$
b c \leq(>) \frac{(3+\sqrt{5}) a^{2}}{2} \Rightarrow \Pi_{S-U S}^{T^{*}}\left(\frac{b c}{a}\right)=\frac{A_{0}^{2} c\left(3 b c-a^{2}\right)}{2\left(2 b c-a^{2}\right)^{2}} \geq(<) \frac{A_{0}^{2}}{2 b}=\Pi_{F-U S}^{T^{*}}(\bar{k}), \text { for any } \bar{k} \text {. }
$$

Therefore, if $b c \leq \frac{(3+\sqrt{5}) a^{2}}{2}$, then $\Pi_{s-U S}^{T^{*}}(\bar{k}) \geq \Pi_{F-U S}^{T^{*}}(\bar{k})$ for all $\bar{k} \in(0, \infty)$. On the other hand, if $440 \quad b c>\frac{(3+\sqrt{5}) a^{2}}{2}$, there exists a unique $\bar{k}_{e}^{\#} \in\left(\bar{k}_{e}^{*}, b c / a\right)$ such that $\Pi_{S-U S}^{T^{*}}(\bar{k}) \geq \Pi_{F-U S}^{T^{*}}(\bar{k})$ for all $\bar{k} \in\left(0, \bar{k}_{e}^{\#}\right]$ and $441 \quad \Pi_{S-U S}^{T^{*}}(\bar{k})<\Pi_{F-U S}^{T^{*}}(\bar{k})$ for all $\bar{k} \in\left(\bar{k}_{e}^{\#}, \infty\right)$. Corollary 1 is thus proved.

442 Corollary 2: There exists a unique $\bar{k}_{e}^{\# \#}>\bar{k}_{e}^{* *}$ such that $\Pi_{s-D S}^{T^{*}}(\bar{k}) \geq \Pi_{F-D S}^{T^{*}}(\bar{k})$ for $\bar{k} \in\left(0, \bar{k}_{e}^{\# \#}\right.$ and $443 \quad \Pi_{S-D S}^{T^{*}}(\bar{k})<\Pi_{F-D S}^{T^{*}}(\bar{k})$ for $\bar{k} \in\left(\bar{k}_{e}^{\# \#}, \infty\right)$.

444 Proof: Since $\Pi_{S-D S}^{T^{*}}(\bar{k})$ reaches its maximum at $\bar{k}_{e}^{* *} \in(a / 2, a)$, then (17) implies that $445 \Pi_{S-D S}^{T^{*}}\left(\bar{k}_{e}^{* * *}\right)>\Pi_{S-D S}^{T^{*}}(a / 2)=\Pi_{F-D S}^{T^{*}}(a / 2)=\Pi_{F-D S}^{T^{*}}(\bar{k})$ for all $\bar{k}>a / 2$. Further, due to $\lim _{\bar{k} \rightarrow \infty} \Pi_{S-D S}^{T^{*}}(\bar{k})=0$ and the 446 unimodality of $\Pi_{S-D S}^{T^{*}}(\bar{k})$, it follows that there is a unique $\bar{k}_{e}^{\# \#}>\bar{k}_{e}^{* *}$ such that $\Pi_{S-D S}^{T^{*}}(\bar{k}) \geq \Pi_{F-D S}^{T^{*}}(\bar{k})$ for all $447 \bar{k} \in\left(0, \bar{k}_{e}^{\# \#}\right)$ and $\Pi_{S-D S}^{T^{*}}(\bar{k})<\Pi_{F-D S}^{T^{*}}(\bar{k})$ for all $\bar{k} \in\left(\bar{k}_{e}^{\# \#}, \infty\right)$. Corollary 2 is then proved.

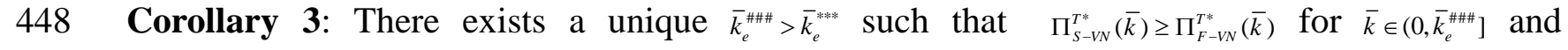


$\Pi_{S-V N}^{T^{*}}(\bar{k})<\Pi_{F-V N}^{T^{*}}(\bar{k})$ for $\bar{k} \in\left(\bar{k}_{e}^{\# \# \#}, \infty\right)$.

Proof: it is trivial to verify that $\lim _{\bar{k} \rightarrow \infty} \Pi_{S-V N}^{T^{*}}(\bar{k})=0$ and $\lim _{\bar{k} \rightarrow 0} \Pi_{S-V N}^{T^{*}}(\bar{k})=A_{0}^{2} /(2 b)=$ $\Pi_{F-V N}^{T^{*}}(\bar{k})$ for all $\bar{k} \in(0, \infty)$. Then the unique maximum of $\Pi_{S-V N}^{T^{*}}(\bar{k})$ at $\bar{k}_{e}^{* * * *} \in(a / 2, \sqrt{b c})$ implies that part (iii) holds. Corollary 3 is thus proved.

453 Remark: Under the basic model setting that CSR performance-related cost incurs only by the 454 supplier, to maximize the channel profit of the supply chain, Proposition 7 indicates that the right to price CSR performance via a wholesale price contract should be allocated to the supplier regardless of the power structure. The corresponding optimal $\bar{k}$ values are derived therein for the three power structures, US, DS, and VN, respectively. When $\bar{k}$ is set at a value other than its optimality, Corollaries 1-3 further reveal a range of values within which it remains optimal to allocate the right to $\mathrm{S}$ for each of the three power structures. Except for the US case with $b c \leq(3+\sqrt{5}) a^{2} / 2$ where it is always better, in terms of system-wide profit, to allocate the right to S, Corollaries 1-3 highlight the importance of placing appropriate caps on $k\left(\bar{k}_{e}^{\#}, \bar{k}_{e}^{\# \#}, \bar{k}_{e}^{\# \#}\right)$ : within these limits, the system-wide profit will be higher if the right is allocated to $S$; once these thresholds are exceeded, it would be better to entrust the right to F. Intuitively, if S's right of pricing CSR performance into a wholesale price contract is not appropriately restricted, it tends to abuse the right by shifting too much cost to F, thereby hurting the overall channel profitability. These results demonstrate that the responsibility holder allocation depends on how to restrict the right by placing a cap on $k$ rather than the power structure within a supply chain. In contrary to the suggestion of Amaeshi et al. (2008) that the more powerful member in a supply chain should be held responsible, Proposition 7 tends to partially support the argument based on the principles of corporate legal personality and separate existence of a corporation that each member is responsible for only its own activity if the right corresponding to the responsibility is appropriately restricted. Note further that given $c_{0}$ (then $w_{0}$ is fixed), the wholesale price $w(y)$ is

473 determined by $k$ and $y$. Therefore, Proposition 7 indicates that, with an appropriate restriction 474 on the right to price CSR performance, the system-wide optimal economic performance can be 475 achieved by allocating the right to the supplier who incurs the investment in social responsibility.

476 It is reasonable to question who controls the allocation right of the contract and how the 477 optimal allocation scheme is implemented. Note that this research assumes that information is 478 complete and symmetric for both parties, and the decision-makers are rational. When the channel 
profit is chosen as the economic criterion for the supply chain, the comparative institutional analysis suggests that the profit maximization drives $\mathrm{S}$ and $\mathrm{F}$ to reach the optimal allocation scheme given in Proposition 7. As for the implementation issue, for the US and VN cases, it is confirmed that $\Pi_{S-U S}^{S^{*}}\left(\bar{k}_{e}^{*}\right) \geq \Pi_{F-U S}^{S^{*}} \quad$ and $\quad \Pi_{S-U S}^{F^{*}}\left(\bar{k}_{e}^{*}\right) \geq \Pi_{F-U S}^{F^{*}} \quad, \quad$ and $\quad \Pi_{S-V N}^{S^{*}}\left(\bar{k}_{e}^{* * *}\right) \geq \Pi_{F-V N}^{S^{*}} \quad$ and $\Pi_{S-V N}^{F^{*}}\left(\bar{k}_{e}^{* * *}\right) \geq \Pi_{F-V N}^{F^{*}}$, indicating that the optimal allocation scheme not only increases the systemwide profit, but also enhances each party’s individual profitability. Therefore, the implementation of the optimal solution is not an issue as it is in the economic interest of each participant in these two cases. On the other hand, in the DS case, we have $\Pi_{S-D S}^{S *}\left(\bar{k}_{e}^{* *}\right)>\Pi_{F-D S}^{S^{*}}$ and $\Pi_{S-D S}^{F^{*}}\left(\bar{k}_{e}^{* *}\right)<\Pi_{F-D S}^{F^{*}}$, indicating that F's profit actually goes down by implementing the optimal allocation scheme although the system-wide profit increases. In this case, due to the complete and symmetric information assumption, an appropriate lump-sum transfer payment from $\mathrm{S}$ to $\mathrm{F}$ exists such that the optimal allocation becomes a win-win solution for both parties. As a matter of fact, let $\Delta$ be the transfer payment, as long as $\Delta>\Pi_{F-D S}^{F^{*}}-\Pi_{S-D S}^{F^{*}}\left(\bar{k}_{e}^{* *}\right)$ and $\left[\Pi_{S-D S}^{S^{*}}\left(\bar{k}_{e}^{* *}\right)-\Pi_{F-D S}^{S^{*}}\right]-\Delta>0$, the optimal allocation of $\mathrm{S}$ being the responsibility holder makes both $\mathrm{S}$ and $\mathrm{F}$ better off. Due to the fact that $\Pi_{S-D S}^{S^{*}}\left(\bar{k}_{e}^{* *}\right)+\Pi_{S-D S}^{F^{*}}\left(\bar{k}_{e}^{* *}\right)>\Pi_{F-D S}^{S^{*}}+\Pi_{F-D S}^{F^{*}}$ as per the argument leading to Proposition 7 , the existence of such a $\Delta$ is guaranteed. Furthermore, the assumption of complete and symmetric information allows for establishing this transfer payment as an enforceable clause of the wholesale contract, which is consistent with the implicit assumption of enforceability based on transfer payments in Hsueh and Chang (2008) as well.

\subsection{Optimal responsibility allocations according to the CSR performance criterion}

In the US case, Propositions 1 and 2 clearly indicate that

$$
y_{S-U S}^{*}(\bar{k})>0=y_{F-U S}^{*}(\bar{k}), \quad \text { for } \bar{k} \in(0, \infty) .
$$

In addition, for all $\bar{k} \in(0, b c / a)$,

$$
\frac{\mathrm{d} y_{s-U S}^{*}}{\mathrm{~d} \bar{k}}=\frac{A_{0}\left(b c-2 \bar{k}^{2}\right)}{[b c-2 \bar{k}(a-\bar{k})]^{2}} .
$$

Then $y_{s-u s}^{*}$ increases for all $\bar{k} \in(0, \sqrt{b c / 2}]$ and decreases for all $\bar{k} \in(\sqrt{b c / 2}, b c / a)$. Thus $y_{s-u s}^{*}$ is unimodal in $\bar{k}$ and attains its maximum at $\bar{k}_{y}^{*}=\sqrt{b c / 2}$ over $(0, b c / a)$. Note that for $\bar{k} \in(b c / a, \infty)$, $y_{s-u s}^{*}(\bar{k})=y_{s-U s}^{*}(b c / a)<y_{s-U s}^{*}\left(\bar{k}_{y}^{*}\right)$, so $y_{s-U s}^{*}$ reaches its global maximum at $\bar{k}_{y}^{*}$. Moreover, (19) implies that 
performance criterion.

In the DS case, part (i) of Proposition 4 indicates that $y_{S-D S}^{*}(\bar{k})=y_{F-D S}^{*}(\bar{k})$ for all $\bar{k} \leq a / 2$. For

$509 \bar{k}>a / 2$, we have

$$
\frac{\mathrm{d} y_{S-D S}^{*}}{\mathrm{~d} \bar{k}}=\frac{A_{0}\left(b c-2 \bar{k}^{2}\right)}{[b c-2 \bar{k}(a-\bar{k})]^{2}} .
$$

511 Then $y_{s-D s}^{*}$ is unimodal in $\bar{k}$ and attains its maximum at $\bar{k}_{y}^{* *}=\bar{k}_{y}^{*}=\sqrt{b c / 2}$. Note that for $\bar{k}>a / 2$, $512 \dot{y}_{F-D S}^{*}(\bar{k})=y_{F-D S}^{*}(a / 2)=y_{s-\text { ss }}^{*}(a / 2)<y_{s-D S}^{*}\left(\bar{k}_{y}^{*}\right)$. Thus $\left(S, \bar{k}_{y}^{*}\right)$ is the unique optimal responsibility allocation 513 according to the CSR performance criterion in the DS case.

514 In the VN case, from Proposition 5 and 6, it follows that for all $\bar{k} \in(0, \infty)$,

$$
y_{S-V N}^{*}(\bar{k})=\frac{A_{0} \bar{k}}{b c-\bar{k}(a-\bar{k})}>0=y_{F-V N}^{*}(\bar{k}),
$$

516 and $y_{F-D S}^{*}(\bar{k})=0<y_{s-V N}^{*}(\bar{k}) \leq y_{s-V N}^{*}\left(\bar{k}_{y}^{* * *}\right)$. Thus $\left(S, \bar{k}_{y}^{*-*}\right)$ is the unique optimal responsibility allocation according to 520 the CSR performance criterion in the $\mathrm{VN}$ structure.

521 Proposition 8: According to the CSR performance criterion, $\left(S, \bar{k}_{y}^{*}\right),\left(S, \bar{k}_{y}^{* *}\right)$ and $\left(S, \bar{k}_{y}^{* *}\right)$ are the 522 unique optimal responsibility allocations in the US, DS, and VN cases, respectively.

523 Corollary 4: (i) For all $\bar{k} \in(0, \infty), y_{S-U S}^{*}(\bar{k})>y_{F-U S}^{*}(\bar{k})$ and $y_{S-T N}^{*}(\bar{k})>y_{F-T N}^{*}(\bar{k})$. (ii) There is a unique $524 \bar{k}_{y}^{*}>\bar{k}_{y}^{* \prime}=\bar{k}_{y}^{*}$ such that $y_{s-D S}^{*}(\bar{k}) \geq y_{F-D S}^{*}(\bar{k})$ for all $\bar{k} \in\left(0, \bar{k}_{y}^{*}\right]$ and $y_{s-D S}^{*}(\bar{k})<y_{F-D S}^{*}(\bar{k})$ for all $\bar{k} \in\left(\bar{k}_{y}^{*}, \infty\right)$.

525 Proof: Part (i) is straightforward. For part (ii), when $\bar{k}>a / 2$, since $\lim _{\bar{k} \rightarrow \infty} y_{s-D s}^{*}(\bar{k})=0$ and $526 y_{F-D S}^{*}(\bar{k})=y_{F-D S}^{*}(a / 2)=y_{s-U s}^{*}(a / 2)<y_{s-D S}^{*}\left(\bar{k}_{y}^{* *}\right)$, then the unimodality of $y_{S-D S}^{*}$ at $\bar{k}_{y}^{* *}$ implies that there is a 527 unique $\bar{k}_{y}^{*}>k_{y}^{* \prime}$ such that $y_{s-D S}^{*}(\bar{k}) \geq y_{F-D S}^{*}(\bar{k})$ for all $\bar{k} \in\left(a / 2, \bar{k}_{y}^{\sharp}\right]$ and $y_{s-D S}^{*}(\bar{k})<y_{F-D S}^{*}(\bar{k})$ for all $\bar{k} \in\left(\bar{k}_{y}^{*}, \infty\right)$. Note 528 further that $y_{s-D S}^{*}(\bar{k})=y_{F-D S}^{*}(\bar{k})$ for all $\bar{k} \leq a / 2$. Then part (ii) is proved. This completes the proof of 529 Corollary 4.

530 Remark: When the objective is to maximize the channel CSR performance, the current model 531 demonstrates that the optimal social responsibility allocation is to designate $S$ as the 532 responsibility holder and entrust it with the (optimally restricted) right to price CSR performance 
533 in a wholesale price contract under each of the three power structures. Corollary 4 further reveals

534 that, even if $\bar{k}$ is not set at its optimality, a higher CSR performance is always achieved by 535 assigning $\mathrm{S}$ as the responsibility holder in the US and VN cases where $\mathrm{S}$ is stronger (US) or 536 equally powerful (VN). But for the DS structure where the downstream $\mathrm{F}$ is more powerful, to 537 make the weaker player $\mathrm{S}$ to be the responsibility holder, an appropriate restriction on the right $538\left(\bar{k}_{y}^{*}\right)$ has to be imposed; otherwise, the more powerful $\mathrm{F}$ will arise as a better choice. Therefore, 539 Corollary 4 is by and large compatible with the suggestion of Amaeshi et al. (2008) that the more 540 powerful player should bear social responsibility.

\subsection{Conflict between the economic and the CSR performance criteria}

542 Due to the uniqueness of $\bar{k}_{y}^{*}, \bar{k}_{y}^{*-*}, \bar{k}_{y}^{-*}, \bar{k}_{e}^{*}, \bar{k}_{e}^{* *}$ and $\bar{k}_{e}^{*-*}$, we show the conflict between the social and 543 economic performance criteria by asserting $\bar{k}_{y}^{*} \neq \bar{k}_{e}^{*}, \bar{k}_{y}^{*-*} \neq \bar{k}_{e}^{*-*}$ and $\bar{k}_{y}^{-\cdots} \neq \bar{k}_{e}^{-\cdots}$.

544 For the US case, as $\bar{k}_{y}^{*}=\sqrt{b c / 2}<b c / a$, substituting $\bar{k}_{y}^{*}=\sqrt{b c / 2}$ into the expression of $\mathrm{d} \Pi_{s-u s}^{T *} / \mathrm{d} \bar{k}$ in 545 (15) yields

$$
\left.\frac{\mathrm{d} \Pi_{S-U S}^{T^{*}}}{\mathrm{~d} \bar{k}}\right|_{\bar{k}=\bar{k}_{y}=\sqrt{b c / 2}}=-\frac{A_{0}^{2} \sqrt{2 b c}}{8 b(\sqrt{2 b c}-a)}<0 .
$$

Given that $\mathrm{d} \Pi_{S-U S}^{T^{*}}\left(\bar{k}_{e}^{*}\right) / \mathrm{d} \bar{k}=0$ and $\Pi_{S-U S}^{T^{*}}(\bar{k})$ reaches its maximum at $\bar{k}_{e}^{*}, \bar{k}_{y}^{*} \neq \bar{k}_{e}^{*}$. Furthermore, the unimodality of $\Pi_{s-U s}^{T^{*}}$ with respect to $\bar{k}$ implies that $\bar{k}_{y}^{*}>\bar{k}_{e}^{*}$.

For the DS and the VN cases, we can similarly ascertain that

551 and

$$
\left.\frac{\mathrm{d} \Pi_{S-D S}^{T^{*}}}{\mathrm{~d} \bar{k}}\right|_{\bar{k}=\bar{k}_{y}^{*}=\sqrt{b c / 2}}=-\frac{A_{0}^{2}}{2 b(\sqrt{2 b c}-a)}<0,
$$

Proposition 9: Assume that $\bar{k}_{e}^{*}, \bar{k}_{e}^{* *}$, and $\bar{k}_{e}^{* * *}$ are the optimal $\bar{k}$ values corresponding to the three power structures as given in Proposition 7 and $\bar{k}_{y}^{*}=\bar{k}_{y}^{* * *}=\sqrt{b c / 2}$, and $\bar{k}_{y}^{*+*}=\sqrt{b c}$ are the optimal $\bar{k}$ values corresponding to the three power structures as given in Proposition 8, then $\bar{k}_{y}^{*}>\bar{k}_{e}^{*}, \bar{k}_{y}^{*}>\bar{k}_{e}^{*}$, and $\overline{k_{y}}>\bar{k}_{e}^{-\cdots}$. 
559 Remark: Propositions 7 and 8 indicate that the optimal economic and CSR performances could 560 be attained by allocating $S$ as the social responsibility holder with appropriate restrictions on $k$ 561 when each criterion is independently considered as a single objective. Proposition 9 further 562 points out that these two criteria are inherently in conflict with each other and it is impossible to 563 achieve both optimality simultaneously under any of the three power structures. In other words, 564 if the economic performance is to be maximized, the channel CSR performance measured by $y$ 565 will not achieve its maximum, and vice versa. Proposition 9 highlights the tradeoff between the 566 economic and CSR performance criteria. This finding sheds significant insights for supply chain managers (the primary member, in particular) who are under increasing pressure for socially 568 responsible business practices: it might well be the case of finding a right trade-off between 569 social and economic performances. Recent research indicates that supply chain managers have started to address consumer confidence and trust about whether goods and services are provided without compromising ethical and environmental standards (New 2003).

\subsection{Comparisons of economic and social responsibility performance}

573 This subsection compares the channel optimal profits, sales quantities, and CSR performance for 574 the decentralized system under the three power structures with those of the integrative case with 575 and without social responsibility considerations. The results are summarized in Proposition 10.

576 Proposition 10: Let $q_{I}^{*}$ and $q_{N}^{*}$ be the optimal sales quantities for the integrative case with and 577 without considering social responsibility, and $\left(q_{S-U S}^{*}\left(\bar{k}_{e}^{*}\right), q_{S-D S}^{*}\left(\bar{k}_{e}^{* * *}\right), q_{S-V N}^{*}\left(\bar{k}_{e}^{* * * * *}\right)\right)$ and $\left(y_{S-U S}^{*}\left(\bar{k}_{e}^{*}\right)\right.$, $\left.578 y_{S-D S}^{*}\left(\bar{k}_{e}^{* * *}\right), y_{S-V N}^{*}\left(\bar{k}_{e}^{* * * *}\right)\right)$ be the optimal quantity and the CSR performance vectors as per the 579 optimal social responsibility allocation schemes for the three power structures as given in 580 Proposition 7. The corresponding profits below are distinguished by their subscripts in a similar 581 fashion. Then

(i) $q_{I}^{*}>q_{s-L s}^{*}\left(\bar{k}_{e}^{*}\right)>q_{N}^{*}, q_{1}^{*}>q_{s-D s}^{*}\left(\bar{k}_{e}^{* *}\right)>q_{N}^{*}$, and $q_{i}^{*}>q_{s-N N}^{*}\left(\bar{k}_{e}^{*-\prime}\right)>q_{N}^{*}$;

(ii) $\Pi_{I}^{*}>\Pi_{S-U S}^{T^{*}}\left(\bar{k}_{e}^{*}\right)>\Pi_{N}^{*}, \Pi_{I}^{*}>\Pi_{S-D S}^{T^{*}}\left(\bar{k}_{e}^{* *}\right)>\Pi_{N}^{*}$, and $\Pi_{I}^{*}>\Pi_{S-V N}^{T^{*}}\left(\bar{k}_{e}^{* * *}\right)>\Pi_{N}^{*}$ and

(iii) If $b c<2 a^{2}$, then $y_{S-U S}^{*}\left(\bar{k}_{e}^{*}\right)<y_{I}^{*}, y_{S-D S}^{*}\left(\bar{k}_{e}^{* * *}\right)<y_{I}^{*}$ and $y_{S-V N}^{*}\left(\bar{k}_{e}^{* * * *}\right)<y_{I}^{*}$.

Proof: For part (i), we only prove that $q_{1}^{*}>q_{s-u s}^{*}\left(\bar{k}_{e}^{*}\right)>q_{N}^{*}$ as the other two cases can be shown in a similar fashion. From part (i) of Proposition 1 , for $\bar{k} \in(0, b c / a]$, we have

$$
q_{I}^{*}>q_{S-U S}^{*}(\bar{k}) \Leftrightarrow W(\bar{k})=a^{2} b c-a\left(a^{2}+b c\right) \bar{k}+\left(a^{2}+b c\right) \bar{k}^{2}>0 .
$$

Since $a^{2}<b c$, we have 


$$
\left[a\left(a^{2}+b c\right)\right]^{2}-4 a^{2} b c\left(a^{2}+b c\right)=-3(a b c)^{2}-2 a^{4} b c+a^{6}=-3(a b c)^{2}+a^{4}\left(a^{2}-2 b c\right)<0 .
$$

Thus the equation $W(\bar{k})=0$ does not have any root over $(0, b c / a]$, and the convexity of $W(\bar{k})$ and $W(0)=a^{2} b c>0$ imply that $W(\bar{k})>0$ for all $\bar{k} \in(0, b c / a]$. For $\bar{k} \in(b c / a, \infty)$, as $q_{I}^{*}=\left(A_{0} c\right) /\left(b c-a^{2}\right)$ and $q_{S-U S}^{*}=\left(A_{0} c\right) /\left(2 b c-a^{2}\right)$ and they share the same numerator with the latter having a larger denominator, it is obvious $q_{I}^{*}>q_{S-U S}^{*}(\bar{k})$. Then $q_{I}^{*}>q_{s-U S}^{*}(\bar{k})$ for all $\bar{k} \in(0, \infty)$. Therefore, $q_{I}^{*}>q_{s-U s}^{*}\left(\bar{k}_{e}^{*}\right)$. Note that $\bar{k}_{e}^{*}<a<b c / a$ as per the proof of Proposition 7, Proposition 1(i) yields

$$
q_{S-U S}^{*}\left(\bar{k}_{e}^{*}\right)=\frac{A_{0}\left[b c-\bar{k}_{e}^{*}\left(a-\bar{k}_{e}^{*}\right)\right]}{b\left[b c-2 \bar{k}_{e}^{*}\left(a-\bar{k}_{e}^{*}\right)\right]}>\frac{A_{0}}{b}=q_{N}^{*} .
$$

For part (ii), for the same reason, we only prove $\Pi_{I}^{*}>\Pi_{s-U S}^{*}\left(\bar{k}_{e}^{*}\right)>\Pi_{N}^{*}$. From (6), $\Pi^{T}(q, y)$ is strictly concave in $(q, y)$. Then $\Pi_{I}^{*}=\Pi^{T}\left(q_{I}^{*}, y_{I}^{*}\right)>\Pi^{T}(q, y)$ for any $(q, y) \neq\left(q_{I}^{*}, y_{I}^{*}\right)$. From part (i), $q_{I}^{*}>q_{s-u s}^{*}\left(\bar{k}_{e}^{*}\right)$, hence $\Pi_{I}^{*}>\Pi_{S-U S}^{*}\left(\bar{k}_{e}^{*}\right)$. Furthermore, the optimality of $\bar{k}_{e}^{*}$ implies that $\Pi_{S-U S}^{*}\left(\bar{k}_{e}^{*}\right)>\Pi_{F-U S}^{*}(\bar{k})=A_{0}^{2} /(2 b)=\Pi_{N}^{*}$.

For part (iii), we first prove $y_{S-U S}^{*}\left(\bar{k}_{e}^{*}\right)<y_{I}^{*}$. Note that

$$
y_{S-U S}^{*}\left(\bar{k}_{e}^{*}\right)<\max _{\bar{k}} y_{S-U S}^{*}(\bar{k})=y_{S-U S}^{*}(\sqrt{b c / 2})=\frac{A_{0}}{2(\sqrt{2 b c}-a)}=\frac{A_{0} a}{2 a(\sqrt{2 b c}-a)}
$$

$$
<\frac{A_{0} a}{2 \sqrt{b c / 2} \sqrt{2 b c}-2 a^{2}}=\frac{A_{0} a}{2\left(b c-a^{2}\right)}<\frac{A_{0} a}{b c-a^{2}}=y_{I}^{*}
$$

601

602

where the first equality is implied in the deduction of Proposition 8, the second and the third inequalities are due to $b c<2 a^{2}$ and $b c>a^{2}$, respectively.

$$
y_{S-D S}^{*}\left(\bar{k}_{e}^{* * *}\right)<y_{I}^{*} \text { can be proved in a similar fashion. Now we prove } y_{S-V N}^{*}\left(\bar{k}_{e}^{* * * *}\right)<y_{I}^{*} \text {. By } b c<2 a^{2} \text {, }
$$
we have $H(a)=-a\left(2 a^{2}-b c\right)<0\left(H(\cdot)\right.$ is introduced in Eq. (18)). Since $H(a / 2)>0, H\left(\bar{k}_{e}^{* * *}\right)=0$, and $H(\cdot)$ is a decreasing function as per the earlier discussions, it is ascertained that $\bar{k}_{e}^{* * * *} \in(a / 2, a)$. Further, the deduction of Proposition 8 implies that $y_{S-V N}^{*}(\bar{k})$ increases in $\bar{k}$ in $(0, \sqrt{b c})$. Since $\bar{k}_{e}^{* * *}<a$, we have

$$
y_{S-V N}^{*}\left(\bar{k}_{e}^{* * *}\right)<y_{S-V N}^{*}(a)=\frac{A_{0} a}{b c}<\frac{A_{0} a}{b c-a^{2}}=y_{I}^{*}
$$

Proposition 10 is thus proved.

Remark: Proposition 10 clearly demonstrates that, with the presence of CSR, the integrative system-wide optimal profit and sales quantity are not attainable via a decentralized system regardless of how CSR is allocated between the two members ( $S$ and F) due to doublemarginalization. Nevertheless, it does point out that the channel profit and sales can be improved 
614 by implementing the optimal social responsibility allocation schemes in the decentralized system 615 compared to the integrative case without considering social responsibility. An intuitive 616 interpretation is that the sales are improved because the market demand curve is shifted upwards 617 by socially responsible activities (Propositions 1, 3 and 5 show that equilibrium $y$ is strictly 618 greater than 0 , while in the case without CSR, $y$ is always equal to 0), leading to a higher 619 system-wide profit. This enhanced profitability, as discussed at the end of Section 4.1, provides a 620 basis for both parties to improve their individual profitability either automatically or via an 621 appropriate credible transfer payment. Proposition 10 thus helps to explain the recent trend in the 622 business world: more and more companies (often primary firms of global supply chains) commit 623 resources to socially and environmentally responsible activities such as establishing and 624 implementing certain codes of conduct as a means to eventually improving their economic 625 performance ${ }^{10}$. And the prediction of efficiency improvement justifies the empirical findings that 626 CSR is positively related to corporate financial performance (Margolis and Walsh 2001; Orlitzky 627 et al. 2003).

628 In the proof of Proposition 10 (iii), the assumption of $b c<2 a^{2}$ is introduced together with $629 b c>a^{2}$. The following arguments are furnished to justify these two assumptions: (1) For a given 630 market demand characterized by $a$ and $b$, the impact of the CSR investment on the supplier's 631 cost should be restricted to a reasonable range (i.e. $a^{2} / b<c<2 a^{2} / b$ ); (2) the upper bound 632 assumption of $b c<2 a^{2}$ ensures that the optimal $\bar{k}$ 's under all three power structures (i.e. $\bar{k}_{e}^{*}, \bar{k}_{e}^{* *}$ 633 and $\bar{k}_{e}^{* * * *}$ ) is less than $a$. As such, by implementing the optimal allocation scheme, the firm's unit 634 profit margin increases in $y$ (as $(a-k) y$ appears in the profit function (4)), leading to the firm's 635 interests in the supplier's CSR investments (otherwise the firm always prefers to $y=0$ because 636 any increase in $y$ will result in a decrease in its unit profit margin).

\section{Discussions}

638 In Section 4, when the optimal allocation decision is considered, it is assumed that the 639 responsibility holder simultaneously determines $k$ along with the other variable. This section 640 examines the case that $k$ is first determined by the responsibility holder and then other decision 641 variables are subsequently decided as per each of the six aforesaid games.

\footnotetext{
${ }^{10}$ For example, Cone/Roper Cause Related Trends Report (1999) shows that nearly 50\% of larger corporations have programs associated with social issues.
} 
643 Section 2, we now modify them by assuming that $k$ is first determined by the responsibility 644 holder, followed by other decision variables. The modified games are denoted as SS-US, FF-US, 645 SS-DS, FF-DS, SS-VN and FF-VN games, where SS and FF indicate that the supplier and the 646 firm are, respectively, assigned as the responsibility holder and decide $k$ prior to other decision 647 variables. Then we change the subscripts of the equilibrium and optimal decision variables in 648 Sections 2-4 in a similar fashion to reflect the corresponding modified scenarios. For example, $649 \Pi_{S S-U S}^{T^{*}}$ and $\Pi_{F F-U S}^{T^{*}}$ represent the equilibrium supply chain system profits of the SS-US and FF-US 650 games, respectively.

According to Zabel's (1970) method, it is easy to check that the equilibrium variables for the SS-US and FF-DS cases are identical to those in the S-US and F-DS cases, respectively. Especially, we have $\Pi_{S S-U S}^{T^{*}}=\Pi_{S-U S}^{T^{*}}$ and $\Pi_{F F-D S}^{T^{*}}=\Pi_{F-D S}^{T^{*}}$ for all $\bar{k}$. Now let us turn to the other four scenarios. First, the equilibrium variables are derived as follows.

(I) The FF-US game:

(i) if $\bar{k} \leq a / 2$, the equilibrium variables are

$$
\begin{gathered}
k_{F F-U S}^{*}=\bar{k} ; y_{F F-U S}^{*}=\frac{A_{0} \bar{k}}{b c-2 \bar{k}(a-\bar{k})} ; q_{F F-U S}^{*}=\frac{A_{0}[b c-\bar{k}(a-\bar{k})]}{b[b c-2 \bar{k}(a-\bar{k})]} ; \\
\Pi_{F F-U S}^{S^{*}}=\frac{A_{0}^{2} \bar{k}^{2}}{2 b[b c-2 \bar{k}(a-\bar{k})]} ; \Pi_{F F-U S}^{F^{*}}=\frac{A_{0}^{2}[b c-\bar{k}(a-\bar{k})]^{2}}{2 b[b c-2 \bar{k}(a-\bar{k})]^{2}} ; \Pi_{F F-U S}^{T^{*}}=\frac{A_{0}^{2}}{2 b}\left[\frac{\bar{k}^{2}}{b c-2 \bar{k}(a-\bar{k})}+\frac{[b c-\bar{k}(a-\bar{k})]^{2}}{[b c-2 \bar{k}(a-\bar{k})]^{2}}\right] .
\end{gathered}
$$

659 (ii) if $\bar{k}>a / 2$, the equilibrium variables are

$$
\begin{gathered}
k_{F F-U S}^{*}=\frac{a}{2} ; y_{F F-U S}^{*}=\frac{A_{0} a}{2 b c-a^{2}} ; q_{F F-U S}^{*}=\frac{A_{0}\left(4 b c-a^{2}\right)}{2 b\left(2 b c-a^{2}\right)} ; \\
\Pi_{F F-U S}^{S^{*}}=\frac{A_{0}^{2} a^{2}}{4 b\left(2 b c-a^{2}\right)} ; \Pi_{F F-U S}^{F^{*}}=\frac{A_{0}^{2}\left(4 b c-a^{2}\right)^{2}}{8 b\left(2 b c-a^{2}\right)^{2}} ; \Pi_{F F-U S}^{T^{*}}=\frac{A_{0}^{2}\left(16 b^{2} c^{2}-4 b c a^{2}-a^{4}\right)}{8 b\left(2 b c-a^{2}\right)^{2}} .
\end{gathered}
$$

662

(II) The SS-DS game:

663

664

(i) if $\bar{k} \leq \sqrt{b c / 2}$, the equilibrium variables are

665

$$
\begin{gathered}
k_{S S-D S}^{*}=\bar{k} ; y_{S S-D S}^{*}=\frac{A_{0} \bar{k}}{b c-2 \bar{k}(a-\bar{k})} ; q_{S S-D S}^{*}=\frac{A_{0} c}{b c-2 \bar{k}(a-\bar{k})} ; \\
\Pi_{S S-D S}^{S^{*}}=\frac{A_{0}^{2} \bar{k}^{2}}{2[b c-2 \bar{k}(a-\bar{k})]^{2}} ; \Pi_{S S-D S}^{F^{*}}=\frac{A_{0}^{2} c}{2[b c-2 \bar{k}(a-\bar{k})]} ; \Pi_{S S-D S}^{T^{*}}=\frac{A_{0}^{2} c\left(b c-2 \bar{k} a+3 \bar{k}^{2}\right)}{2[b c-2 \bar{k}(a-\bar{k})]^{2}} .
\end{gathered}
$$

666

(ii) if $\bar{k}>\sqrt{b c / 2}$, the equilibrium variables are 


$$
k_{S S-D S}^{*}=\sqrt{\frac{b c}{2}} ; y_{S S-D S}^{*}=\frac{A_{0}}{2(\sqrt{2 b c}-a)} ; q_{S S-D S}^{*}=\frac{A_{0} c}{\sqrt{2 b c}(\sqrt{2 b c}-a)} ;
$$

$$
\Pi_{S S-D S}^{S^{*}}=\frac{A_{0}^{2} c}{8(\sqrt{2 b c}-a)^{2}} ; \Pi_{S S-D S}^{F^{*}}=\frac{A_{0}^{2} c}{2 \sqrt{2 b c}(\sqrt{2 b c}-a)} ; \Pi_{S S-D S}^{T^{*}}=\frac{A_{0}^{2}(5 b c-2 a \sqrt{2 b c})}{8 b(\sqrt{2 b c}-a)^{2}} .
$$

669

(III) The SS-VN game:

670

(i) if $\bar{k} \leq \sqrt{b c}$, the equilibrium variables are

$$
\begin{gathered}
k_{S S-V N}^{*}=\bar{k} ; y_{S S-V N}^{*}=\frac{A_{0} \bar{k}}{b c-\bar{k}(a-\bar{k})} ; q_{S S-V N}^{*}=\frac{A_{0} c}{b c-\bar{k}(a-\bar{k})} ; \\
\Pi_{S S-V N}^{S^{*}}=\frac{A_{0}^{2} \bar{k}^{2}}{2[b c-\bar{k}(a-\bar{k})]^{2}} ; \Pi_{S S-V N}^{F^{*}}=\frac{A_{0}^{2} c}{2[b c-\bar{k}(a-\bar{k})]} ; \Pi_{S S-V N}^{T *}=\frac{A_{0}^{2} c\left(b c-\bar{k} a+2 \bar{k}^{2}\right)}{2[b c-\bar{k}(a-\bar{k})]^{2}} .
\end{gathered}
$$

(ii) if $\bar{k}>\sqrt{b c}$, the equilibrium variables are

676

(IV) The FF-VN game:

(i) if $\bar{k} \leq a / 2$, the equilibrium variables are

$$
\begin{gathered}
k_{F F-V N}^{*}=\bar{k} ; y_{F F-V N}^{*}=\frac{A_{0} \bar{k}}{b c-\bar{k}(a-\bar{k})} ; q_{F F-V N}^{*}=\frac{A_{0} c}{b c-\bar{k}(a-\bar{k})} ; \\
\Pi_{F F-V N}^{S^{*}}=\frac{A_{0}^{2} c \bar{k}^{2}}{2[b c-\bar{k}(a-\bar{k})]^{2}} ; \Pi_{F F-V N}^{F^{*}}=\frac{A_{0}^{2} c}{2[b c-\bar{k}(a-\bar{k})]} ; \Pi_{F F-V N}^{T^{*}}=\frac{A_{0}^{2} c\left(b c-\bar{k} a+2 \bar{k}^{2}\right)}{2[b c-\bar{k}(a-\bar{k})]^{2}} .
\end{gathered}
$$

(ii) if $\bar{k}>a / 2$, the equilibrium variables are

684 Proposition 11 as follows.

685 Proposition 11: For the modified games where $k$ is determined by the responsibility holder 686 before the other decision variables $q$ and $y$ are furnished by F and S, respectively, it remains true 687 for the optimal responsibility allocation schemes derived in Propositions 7 and 8 under each of 688 the three power structures as well as the comparative statics established in Propositions 9 and 10. 689 Proof: We first verify Proposition 7. For the FF-US game, we have 


$$
\Pi_{F F-U S}^{T^{*}}=\left\{\begin{array}{l}
\frac{A_{0}^{2}}{2 b}\left[\frac{\bar{k}^{2}}{b c-2 \bar{k}(a-\bar{k})}+\frac{[b c-\bar{k}(a-\bar{k})]^{2}}{[b c-2 \bar{k}(a-\bar{k})]^{2}}\right], \quad \bar{k} \leq \frac{a}{2} \\
\frac{A_{0}^{2}\left(16 b^{2} c^{2}-4 b c a^{2}-a^{4}\right)}{8 b\left(2 b c-a^{2}\right)^{2}}, \bar{k}>\frac{a}{2}
\end{array} .\right.
$$

Clearly, $\Pi_{F F-U S}^{T^{*}}(\bar{k})=\Pi_{S-U S}^{T^{*}}(\bar{k})=\Pi_{S S-U S}^{T^{*}}(\bar{k})$ for all $\bar{k} \leq a / 2 \leq a \leq b c / a$. Recall that $F(\bar{k})=-2 a \bar{k}^{4}+$

$6922 a^{2} \bar{k}^{3}-b c\left(a^{2}+b c\right) \bar{k}+a b^{2} c^{2}$ is introduced in analyzing the US case in Section 4.1, it is easy to 693 verify that $F(a / 2)=a b c\left(b c-a^{2}\right) / 2>0$. Then $\Pi_{s-U s}^{T^{*}}(\bar{k})$ increases at $\bar{k}=a / 2$. By the definition of $\bar{k}_{e}^{*}$

694 in Section 4.1, we have $\Pi_{S-U S}^{T^{*}}\left(\bar{k}_{e}^{*}\right)>\Pi_{S-U S}^{T^{*}}(a / 2)=\Pi_{F F-U S}^{T^{*}}(a / 2) \geq \Pi_{F F-U S}^{T^{*}}(\bar{k})$, where the last inequality 695 is derived due to the fact that the supply chain system profit function (20) increases over $(0, a / 2)$ 696 and remains constant for $\bar{k} \in[a / 2, \infty)$. Thus, $\left(S, \bar{k}_{e}^{*}\right)$ remains the optimal responsibility allocation 697 for the US case according to the economic performance criterion, even if $k$ is first decided by 698 the firm. In a similar way, we can also confirm that for all $\bar{k}$,

$$
\Pi_{S-D S}^{T^{*}}\left(\bar{k}_{e}^{* * *}\right)=\Pi_{S S-D S}^{T^{* *}}\left(\bar{k}_{e}^{* * *}\right) \geq \Pi_{S S-D S}^{T^{*}}(\bar{k}),
$$

$$
\Pi_{S-V N}^{T^{*}}\left(\bar{k}_{e}^{* * * *}\right)=\Pi_{S S-V N}^{T^{*}}\left(\bar{k}_{e}^{* * * *}\right) \geq \Pi_{S S-V N}^{T^{*}}(\bar{k}) \text { and } \Pi_{S-V N}^{T *}\left(\bar{k}_{e}^{* * * *}\right)=\prod_{F F-V N}^{T^{*}}(a / 2) \geq \Pi_{F F-V N}^{T *}(\bar{k}) \text {. }
$$

(21) and (22) imply that $\left(S, \bar{k}_{e}^{* * *}\right)$ and $\left(S, \bar{k}_{e}^{*+* *}\right)$ are the optimal responsibility allocations for the DS and VN cases, respectively.

Now we prove that Proposition 8 remains true. We can easily determine that for all $\bar{k}$,

$$
y_{S-U S}^{*}\left(\bar{k}_{y}^{*}\right)>y_{S-U S}^{*}(a / 2)=y_{F F-U S}^{*}(a / 2) \geq y_{F F-U S}^{*}(\bar{k}),
$$

$$
y_{S-D S}^{*}\left(\bar{k}_{y}^{* *}\right)=y_{S S-D S}^{*}\left(\bar{k}_{y}^{* *}\right) \geq y_{S S-D S}^{*}(\bar{k}),
$$

$$
y_{S-V N}^{*}\left(\bar{k}_{y}^{* * *}\right)=y_{S S-V N}^{*}\left(\bar{k}_{y}^{* * *}\right) \geq y_{S S-V N}^{*}(\bar{k}) \text { and } y_{S-V N}^{*}\left(\bar{k}_{y}^{* * * *}\right)>y_{F F-V N}^{*}(a / 2) \geq y_{F F-V N}^{*}(\bar{k}) \text {. }
$$

Then (23), (24) and (25) imply that $\left(S, \bar{k}_{y}^{*}\right),\left(S, \bar{k}_{y}^{* *}\right)$ and $\left(S, \bar{k}_{y}^{* * * *}\right)$ are the optimal responsibility allocations for the US, DS and VN cases, respectively.

Finally, since the assumption that $k$ is first decided by the corresponding responsibility holder does not have any impact on the optimal responsibility allocations, Propositions 9 and 10 follow immediately. Proposition 11 is thus proved.

Remark: Two points are worth mentioning here. First, as the responsibility holder's $k$ decision induces the subsequent US, DS or VN game, it has to take into account the subsequent equilibrium variables due to backward induction. This consideration helps to avoid the extreme case of not sharing the CSR investment at all. Second, Proposition 11 shows that Propositions 7- 
71610 are robust to the change of the sequence of determining $k$ as long as $\bar{k}$ is appropriately 717 specified.

718 To illustrate the shapes of and relationships among the channel profit functions, a numerical 719 example has been developed for the US case as shown in Table 1, and the resulting graph is 720 depicted in Fig. 1 (As a matter of fact, the relative relationships among the curves in Fig. 1 can

721 be theoretically confirmed). Fig. 1 clearly points out the optimal allocation at $\bar{k}_{e}^{*}$ (Proposition 722 11). If $\bar{k}$ is not set at its optimality $\bar{k}_{e}^{*}$ and $k$ is offered by the responsibility holder ahead of the 723 other two decision variables, $q$ and $y$, Fig. 1 also furnishes the ranges of $\bar{k}$ values within which 724 the profit is indifferent $(0<\bar{k} \leq a / 2)$, the channel profit is higher if $\mathrm{S}$ is the responsibility holder $725\left(a / 2<\bar{k}<\bar{k}_{e}^{\prime}\right)$, or it is better to entrust $\mathrm{F}$ as the responsibility holder $\left(\bar{k}>\bar{k}_{e}^{\prime}\right)$. Fig. 1 also 726 schematically confirms Proposition 7 and Corollary 1 when $\bar{k}$ is not set at its optimality and $k$ is 727 determined with the responsibility holder's other decision variable simultaneously. For the DS 728 and VN cases, similar numerical experiments and graphical representations can be obtained and 729 are omitted here for the sake of space.

Table 1. Channel profit for the S-US (SS-US), F-US, FF-US cases

\begin{tabular}{|c|c|c|c|c|c|c|}
\hline \multirow{2}{*}{$k$} & \multicolumn{3}{|c|}{$b c>(3+\sqrt{5}) a / 2$} & \multicolumn{3}{c|}{$b c \leq(3+\sqrt{5}) a / 2$} \\
\cline { 2 - 7 } & $\Pi_{S-U S}^{T^{*}}\left(\Pi_{S S-U S}^{T^{*}}\right)$ & $\Pi_{F-U S}^{T^{*}}$ & $\Pi_{F F-U S}^{T^{*}}$ & $\Pi_{S-U S}^{T^{*}}\left(\Pi_{S S-U S}^{T^{*}}\right)$ & $\Pi_{F-U S}^{T^{*}}$ & $\Pi_{F F-U S}^{T^{*}}$ \\
\hline 0 & 0.25 & 0.25 & 0.25 & 0.333333 & 0.333333 & 0.333333 \\
\hline 0.3 & 0.309299 & 0.25 & 0.309299 & 0.503344 & 0.333333 & 0.503344 \\
\hline 0.6 & 0.336389 & 0.25 & 0.323696 & 0.626298 & 0.333333 & 0.604167 \\
\hline 0.9 & 0.322674 & 0.25 & 0.323696 & 0.584883 & 0.333333 & 0.604167 \\
\hline 1.2 & 0.297115 & 0.25 & 0.323696 & 0.499847 & 0.333333 & 0.604167 \\
\hline 1.5 & 0.275543 & 0.25 & 0.323696 & 0.4375 & 0.333333 & 0.604167 \\
\hline 1.8 & 0.259758 & 0.25 & 0.323696 & 0.4375 & 0.333333 & 0.604167 \\
\hline 2.1 & 0.248293 & 0.25 & 0.323696 & 0.4375 & 0.333333 & 0.604167 \\
\hline 2.4 & 0.239755 & 0.25 & 0.323696 & 0.4375 & 0.333333 & 0.604167 \\
\hline 2.7 & 0.237387 & 0.25 & 0.323696 & 0.4375 & 0.333333 & 0.604167 \\
\hline 3.0 & 0.237387 & 0.25 & 0.323696 & 0.4375 & 0.333333 & 0.604167 \\
\hline
\end{tabular}

\footnotetext{
Parameter values are set as follows: $w_{0}=c_{0}=1, a_{0}=2, A_{0}=a_{0}-w_{0}=1$;
} 
732 For the $b c>(3+\sqrt{5}) a / 2$ case, $a=0.8, b=2, c=1$; For the $b c \leq(3+\sqrt{5}) a / 2$ case, $a=1, b=1.5, c=1$.

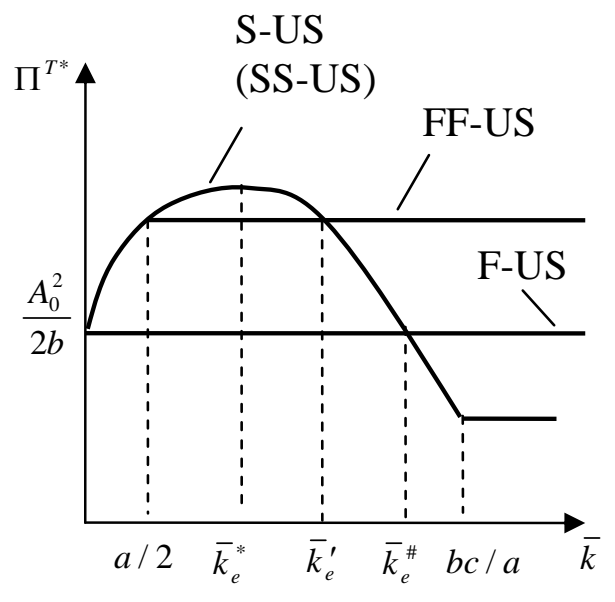

Case (i): $b c>(3+\sqrt{5}) a / 2$

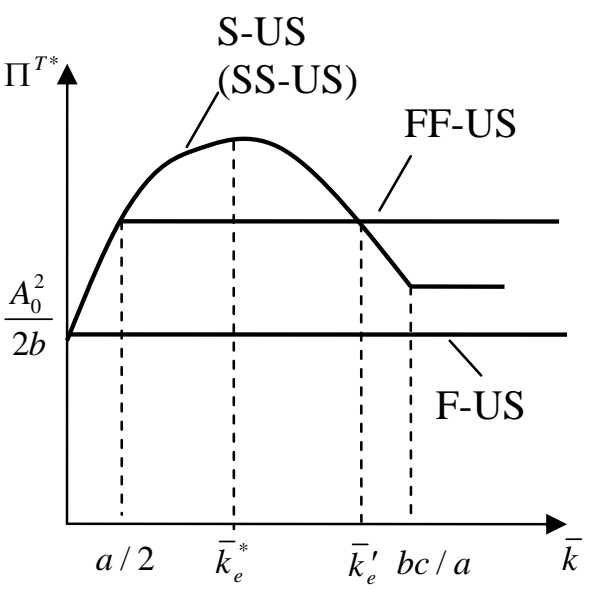

Case (ii): $b c \leq(3+\sqrt{5}) a / 2$

Fig. 1 The Comparison of equilibrium profits (the US case)

\section{Concluding Remarks}

735 Social responsibility allocation is considered in a two-echelon supply chain, consisting of a 736 downstream firm F and an upstream supplier S bound by a wholesale price contract. The CSR 737 performance of the supply chain is assumed to be a global variable $y$ and the related cost is 738 incurred only by $\mathrm{S}$ and is expected to be shared with $\mathrm{F}$ via the wholesale price contract that is 739 characterized by a parameter $k$. With the duality of responsibility and rights, the allocation is 740 conceived as a two-dimensional vector. The first dimension assigns a supply chain member as 741 the responsibility holder and entrusts it with the right to price the CSR performance in the 742 contract. The second dimension specifies an upper bound $\bar{k}$ for the key parameter $k$ in the 743 wholesale price contract, which effectively places a restriction on the right of the responsibility

744 holder. The power structure of the supply chain is captured as the Stackelberg leader-follower 745 relationship. Different combinations of responsibility holder assignment and power structures 746 lead to six distinct games and their corresponding equilibriums are derived accordingly in

747 Propositions 1 through 6. By analyzing the equilibriums as per the methodology of comparative 748 institutional analysis, the following key results are obtained:

(1) Under each of the three power structures, the optimal social responsibility allocation

750 scheme is always to assign the supplier as the responsibility holder with appropriate restrictions 751 on $k$ based on both the economic and the CSR performance criteria (Propositions 7 and 8). 752 When the economic performance drifts away from its maximum, such restrictions are mandatory 
753 for the supplier to be the responsible holder under all the three power structures with a minor 754 exception (Corollaries 1-3). Otherwise, if the cap on $k$ is set at a level that exceeds a threshold, F will turn out to be a better responsibility holder for the channel profit. In the model setting in this research, the investment for ensuring the global social performance y incurs by S only. Therefore, the optimal allocation scheme of entrusting $S$ with the right of pricing $y$ tends to support arguments based on the principles of corporate legal personality and separate existence of a corporation. On the other hand, if the maximal CSR performance is not attained, to make the supplier a better responsibility holder, an appropriate restriction on the right is only required for the DS case when the supplier is a relatively weaker player (Corollary 4). From this perspective, this result is compatible with the suggestion of Amaeshi et al. (2008) that more powerful members should be held accountable.

(2) Under all the three power structures, it is impossible to achieve optimal economic and CSR performance simultaneously. Inherent conflict exists between these two criteria when social responsibility allocation decisions are made (Proposition 9). This result highlights the need for finding an appropriate tradeoff between these two criteria for supply chain managers who are faced with increasing social responsibility pressures in practice, as observed by New (2003).

(3) Under all the three power structures, the integrative channel profit is not attainable due to double-marginalization, but the system-wide profit will be improved by implementing optimal social responsibility allocation schemes compared to the case without considering social responsibility at all (Proposition 10). This result helps us understand the recent trend of investing in social responsibility in the business world, and justifies the empirical findings that CSR is positively related to corporate financial performance (Margolis and Walsh 2001; Orlitzky et al. 2003).

Finally, Proposition 11 shows that Propositions 7-10 are robust relative to the sequence change of determining $k$ (i.e., $k$ is first offered by the responsibility holder) as long as $\bar{k}$ is appropriately specified.

The current model assumes that information on both the cost parameter of social responsibility investment and the parameter of the market impact of CSR performance is symmetric for the supplier and the firm. In reality, the supplier may possess more information on the cost parameter while the firm is likely to understand the market impact better. This information asymmetry raises a new question: How do moral hazard and/or adverse selection 
784 influence social responsibility allocation? Another potential extension of this research is to 785 consider other well-known contract structures such as the buy back contract, the revenue sharing 786 contract, the quantity flexibility contract, to name a few. Still another consideration is to explore 787 the situation that both the supplier and firm incur their individual CSR costs. These open 788 questions warrant further investigations.

\section{Acknowledgements}

790 The authors wish to express their sincere appreciation to the three anonymous reviewers for their 791 constructive comments that significantly improve the quality and presentation of the paper. 792 Debing Ni would like to acknowledge the financial support from the Natural Sciences 793 Foundation (NSF) of China (Grant \#: 70702025) and the UESTC Funds for Junior Research 794 Chairs. Debing Ni and Xiaowo Tang would like to acknowledge the financial support from the 795 Key Project of Natural Sciences Foundation (NSF) of China (Grant \#: 70932005). Kevin W. Li is 796 grateful for the financial support from the Natural Sciences and Engineering Research Council of 797 Canada (NSERC) under the Discovery Grant program.

\section{References}

800 [1] Amaeshi, K., Osuji, O. K. and Nnodim, P. Corporate social responsibility in supply chains of global brands: A boundaryless responsibility? Clarifications, exceptions and implications, Journal of Business Ethics, 2008, 81(1), 223-234.

[2] Baron, D. P. Private politics, corporate social responsibility, and integrated strategy, Journal of Economics \& Management Strategy, 2001, 10(1), 7-45.

[3] Baron, D. P. Corporate social responsibility and social entrepreneurship, Journal of Economics \& Management Strategy, 2007, 16(3), 683-717.

808 [5] Cachon, G. P. Supply chain coordination with contracts, In A. G. de Kok, S. C. Graves, eds., 809 Supply Chain Management: Design, Coordination and Operation (Handbooks in $810 \quad$ Operations Research and Management Science series), Elsevier, 2003.

811 [6] Calveras, A., Ganuza, J.-J., and Llobet, G. Regulation, corporate social responsibility and activism, Journal of Economics \& Management Strategy, 2007, 16(3), 719-740

813 [7] Carter, C. R., Kale, R. and Grimm, C. M. Environmental purchasing and firm performance: An empirical investigation, Transportation Research E, 2000, 36(3), 219-228 
815 [8] Carter, C. R. and Jennings, M. M. Social responsibility and supply chain relationships, 816 Transportation Research E, 2002, 38(1), 37-52

817 [9] Choi, S. C. Price competition in a channel structure with common retailer, Marketing 818 Science, 1991, 10(4), 271-296

819 [10] Coase, R. The problem of social cost, Journal of Law and Economics, 1960, 3: 1-44

820 [11] Cone/Roper Cause Related Trends Report. The Evolution of Cause Branding, Cone, Inc., $821 \quad$ Boston, 1999

822 [12] Crutz, J. M. Dynamics of supply chain networks with corporate social responsibility through integrated environmental decision-making, European Journal of Operational Research, 2008, 184(3), 1005-1031

[13] Davis, G. A., Wilt, C. A. and Barkenbus, J. N. Extended product responsibility: A tool for a sustainable economy, Environment, 1997, 39(7): 10-15, 36-37

[14] Freeman, R. E. Strategic Management: A Stakeholder Approach, Pitman/ Ballinger, Boston, 1984

[15] Giovanni, C. and Giacinta, C. Corporate social responsibility and managerial entrenchment, Journal of Economics \& Management Strategy, 2007, 16(3), 741-771

[16] Gurnani, H., Erkoc, M.and Luo, Y.. Impact of product and timing of investment decisions on supply chain co-opetition. European Journal of operational Research, 2007, 180(1), 224-248

[17] Hsueh, C.-F. and Chang, M.-S. Equilibrium analysis and corporate social responsibility for

[19] Lee, H. L., Duda, S., James, L., Mackwani, Z., Munoz, R. and Volk, D. Building a supply chain integration, European Journal of Operational Research, 2008, 190 (1), 116129

[18] Kwoka, J. E. Vertical economies in electric power: evidence on integration and its alternatives, International Journal of Industrial Organization, 2002, 20 (5), 653-671

843 [20] Linton, JD, Klassen R, Jayaraman, V. (2007), Sustainable supply chains: an introduction. 844 Journal of Operations Management, 25: 1075-1082.

845 [21] Mamic, I. Managing global supply chain: The sports footwear, apparel and retail sectors, 
Journal of Business Ethics, 2005, 59(1/2), 81-100

[22] Margolis, J. D. and Walsh, J. P. People and Profits? The Search for a Link between a Company's Social and Financial Performance, Erlbaum, Mahwah, 2001.

[23] Merrick, GAP offers unusual look at factory conditions: Fighting 'sweatshop' tag retailer detail problems among thousands of plants, Wall Street Journal, 2004, May 12, A1

[24] Mohr, L. A. and Webb, D. J. The effects of corporate social responsibility and price on consumer responses, Journal of Consumer Affairs, 2005, 39(1): 121-147

853 [25] New, S. There may be troubles ahead, Supply Management, 2003, 8(1): 16-20

854 [26] OECD. Extended Producer Responsibility: A guidance Manual for Governments, 2001

855 [27] Orlitzky M., Schmidt, F. L. and Rynes S. L. Corporate social and financial performance: A mete-analysis, Organization Studies, 2003, 24(3): 403-441

[28] Perry, M. K. and Porter, R. H. Oligopoly and the incentive for horizontal merger, American Economic Review, 1985, 75, (1), 219-227

[29] Rath, K. P. and Zhao, G. Two stage equilibrium and product choice with elastic demand, International Journal of Industrial Organization, 2001, 19 (9) 1441-1455

[30] Röller, L.-H. Proper quadratic cost functions with an application to the bell system, Review of Economics and Statistics, 1990, 72(2), 202-210

[31] Savaskan, R. C., Bhattacharya, S. and Van Wassenhove, L. N. Closed-loop supply chain

[34] World Bank, Strengthening implementation of corporate social responsibility in global supply chains, Washington D. C. 2003

[35] Xiao, T. and Yang, Q. Price and service competition of supply chains with risk-averse retailers under demand uncertainty. International Journal of Production Economics, 2008, 114(1), 187-200

[36] Zabel, E. Monopoly and uncertainty, Review of Economic Studies, 1970, 37(2), 205-219 